\title{
Closing the gap: accelerating the translational process in nanomedicine by proposing standardized characterization techniques
}

This article was published in the following Dove Press journal:

International Journal of Nanomedicine

8 December 2014

Number of times this article has been viewed

\author{
Ali A Khorasani ${ }^{1-3}$ \\ James L Weaver ${ }^{4}$ \\ Carolina Salvador-Morales ${ }^{2,3}$ \\ 'Department of Chemistry and \\ Biochemistry, ${ }^{2}$ Bioengineering \\ Department, ${ }^{3}$ Krasnow Institute \\ for Advanced Study, George Mason \\ University, Fairfax, VA, USA; ${ }^{4}$ Division \\ of Applied Regulatory Science, Center \\ for Drug Evaluation and Research, \\ Food and Drug Administration, Silver \\ Spring, MD, USA
}

\begin{abstract}
On the cusp of widespread permeation of nanomedicine, academia, industry, and government have invested substantial financial resources in developing new ways to better treat diseases. Materials have unique physical and chemical properties at the nanoscale compared with their bulk or small-molecule analogs. These unique properties have been greatly advantageous in providing innovative solutions for medical treatments at the bench level. However, nanomedicine research has not yet fully permeated the clinical setting because of several limitations. Among these limitations are the lack of universal standards for characterizing nanomaterials and the limited knowledge that we possess regarding the interactions between nanomaterials and biological entities such as proteins. In this review, we report on recent developments in the characterization of nanomaterials as well as the newest information about the interactions between nanomaterials and proteins in the human body. We propose a standard set of techniques for universal characterization of nanomaterials. We also address relevant regulatory issues involved in the translational process for the development of drug molecules and drug delivery systems. Adherence and refinement of a universal standard in nanomaterial characterization as well as the acquisition of a deeper understanding of nanomaterials and proteins will likely accelerate the use of nanomedicine in common practice to a great extent.
\end{abstract}

Keywords: physicochemical characterization, poly(ethylene glycol), protein adsorption, regulatory issues

\section{Introduction}

There is a prevailing consensus in the scientific literature that nanotechnology has already permeated a variety of applications ranging from medicine to energy to electronics. ${ }^{1}$ Nanomaterials certainly present unique solutions to medical problems due to their unique nanoscale size. However, biomedical research in general and nanomedicine in particular are limited by a translational gap between experimental work and practical applications, thus hindering the expected nanomedicine boom.

The US government believes that nanotechnology will shape the economic development of the country because of its impact in the key areas of medicine, energy, and electronics. As such, the US National Nanotechnology Initiative, which includes a number of government departments working together to invest in nanotechnology research, has a 2015 proposed annual budget of $\$ 1.54$ billion. ${ }^{1}$ The National Science Foundation provides $\$ 412.4$ million of funding in this area, and the US Food and Drug Administration (FDA) proposed \$17.0 million in its FY2015 budget as a part of the National Nanotechnology Initiative. ${ }^{1}$ Similar programs in the European Union, Japan, the People's Republic of China, Taiwan, and Korea spent a total of $\$ 3.5$ billion annually. ${ }^{2}$ This spending reflects the governments' recognition of the need
Correspondence: Carolina SalvadorMorales

Department of Bioengineering, George Mason University, 4400 University Drive MS IG5, Fairfax, VA 22030, USA

Tel +l 7039935895

Fax + I 7039932077

Email csalvado@gmu.edu (c) (i) (9) 2014 Khorasani et al. This work is published by Dove Medical Press Limited, and licensed under Creative Commons Attribution - Non Commercial (unported, v3.0) License. The full terms of the License are available at http://creativecommons.org/licenses/by-nc/3.0/. Non-commercial uses of the work are permitted without any further permision how to request permission may be found at: http://www.dovepress.com/permissions.php 
to further understand the safe and effective use of nanomaterials, but does not directly address improving the process by which nanoproducts are developed and brought to commercial therapeutic use. Nanomaterials must be able to fully comply with the rigorous standards required by regulatory agencies in order to transition into everyday use.

Some commonly approved types of materials employed in nanomedicine research include polymeric and metallic nanoparticles, which are the favorite candidates for research and industry studies because of their potential utility to improve medical treatments in a wide range of diseases. Metallic particles are widely proposed for use as diagnostic tools because they provide unique contrast with the mostly organic matter in biological systems. As the diagnostic industry rapidly increases, more and more opportunities arise for using metallic nanoparticles to develop methods that are more sensitive and specific for biomarker detection in different body fluids, thereby bridging the translational gap. However, as metallic, polymeric, and other types of nanoparticles with increasing complexity and functionality are developed, it becomes very important to have precise control of the functionality, uniformity, and dispersion of these nanomaterials. Traditional means used for physicochemical characterization with small-molecule or bulk systems are often hampered by the unique constraints of the nanoscale, which makes it difficult to universally characterize different nanoparticle systems. Therefore, it is important to develop standardized processes and techniques for nanoparticle characterization in order to overcome the translational gap, and nanomaterials must be consistently and verifiably manufactured in order to ensure quality and effectiveness. A number of characterization methods, including the newest innovations to common techniques, will be addressed in this review. We also include a suggested standard set of characterization techniques. Beyond consistency in synthesis, nanomaterials must be safe for use in common medical routes of administration. Intravenous administration is a preferred route for polymeric particles, and most of the nanoproducts under development are intended to be administered intravenously. In the case of tumors, when nanoparticles are administered in this fashion, they can easily act upon the tumors via the passive or active enhanced permeability and retention effect, in which the nanocarrier reaches the tumor because of the leaky tumor vasculature. ${ }^{3}$ Intravenous administration also ensures that the therapeutic effect can be induced faster than other administration routes. If the nanocarrier is administered intravenously, the immunotoxicity of the nanocarrier must be addressed in depth. Given the therapeutic and translational importance of this administration route, the immunocompatibility aspects of nanocarriers will be addressed in this review, emphasizing the interaction between nanomaterials and proteins.

\section{Smarter characterization of nanomaterials}

\section{Limitations on current physicochemical characterization techniques and recent developments in this subfield}

Nanomaterials can present heterogeneous physicochemical properties (such as size, weight, surface area, surface chemistry) to a much greater extent than small-molecule compounds. As aggregates of smaller materials, it is difficult to precisely control the exact dimensions of the particles due to limitations in synthetic methods. Aside from higher-cost synthetic routes that allow for precise control of the surface chemistry of the particle, like photolithography, many synthetic methods rely on the physicochemical characteristics of the parent compounds and affiliated reagents in order to attain a particular physicochemical state. Polymers in particular have inherent molecular weight polydispersity that likely transfers its properties to the nanocarrier. ${ }^{4}$ Therefore, it is clear that characterization of the physicochemical features of a nanomaterial is essential to validate its medical performance. Physicochemical characterization includes physical, chemical, composition, identification, quality, purity, and stability analyses of the nanomaterial. ${ }^{5}$ Although today there are no standards formally established in the scientific community for physicochemical characterization of a nanomaterial, there is an informal scientific consensus that nanomaterials should be characterized in terms of their size distribution, zeta potential, targeting/drug/imaging quantification, purity, stability, and batch-to-batch consistency before conducting in vitro experiments that involve biological entities.

Physicochemical characterization of a nanomaterial can be conducted using a wide range of techniques including dynamic light scattering (DLS), microscopy, spectroscopy, chromatography, and electrochemistry. ${ }^{5}$ The size of a nanocarrier is one of the most important physical characteristics of a nanomaterial, so should be carefully and appropriately measured. The appropriate instruments and methods designed to measure the size of the nanocarrier will depend heavily on its function. DLS has been considered to be the most suitable method for measuring the size of a nanocarrier because it allows us to measure the hydrodynamic diameter of the particle. Other instruments, such as the transmission electron microscope (TEM), have 
been used to measure nanoparticle size; however, the size of the nanocarrier obtained by TEM is not accurate, given that it should be measured in aqueous solution, ie, the physiological environment in which the nanocarrier will perform its function.

DLS-derived particle counts, representing the statistical polydispersity of synthetic nanomaterials, can be quantified in various ways, eg, averaging the size of the particle based on intensity, volume, or z-number. A chapter in a recent book published by the National Characterization Laboratory discusses different aspects of nanoparticle characterization. ${ }^{5}$ It recommends that the intensity-weighted distribution should be used for reporting size because volume-weighted or numberweighted distributions are prone to presenting the data differently. Number-weighted distributions are much narrower, while volume distributions are much wider than intensity-based distribution. ${ }^{5}$ In addition to the DLS method, the small angle $\mathrm{X}$-ray scattering (SAXS) technique has recently been used to measure particle size as well as to elucidate particle shape. ${ }^{6}$

Another important characteristic of the nanocarrier that must be carefully measured is the zeta potential. The zeta potential is defined as the potential difference between the bulk solution (dispersing medium) and the surface of hydrodynamic shear (slipping plane). ${ }^{5}$ Although many research laboratories determine the zeta potential of the nanocarrier using a zetasizer, the parameters used to make zeta potential measurements vary from laboratory to laboratory and are often not reported in research papers. Revealing these parameters is crucial to improve the characterization process of nanomaterials because it will help us to compare results from laboratory to laboratory and from sample to sample. Full disclosure of such parameters would allow researchers to start creating a database that can be used later to identify the best parameters to use when taking the zeta potential of a nanocarrier. While taking zeta potential measurements, it is important to remember that an applied voltage can potentially degrade the nanoparticles being measured, so care needs to be taken when characterizing the materials with other physicochemical characterization methods before and after zetasizer measurement. The accuracy of zeta potential measurements depends on the sample preparation and measurement procedures used. ${ }^{5}$ Guidelines on these important issues are described in detail in the second chapter of the "Handbook of Immunological Properties of Engineered Nanomaterials". 5

The size and zeta potential of the nanocarrier determine its specific biodistribution profile. Almost all administration routes available for nanocarriers require them to be in aqueous solution; hence, it is imperative to determine the hydrodynamic size of a nanocarrier, which changes once it is in the bloodstream due to the phenomenon of adsorption and desorption of proteins. ${ }^{7}$ Consequently, physicochemical characterization of the nanocarrier before, during, and after administration is essential. A summary of the key physicochemical characterization parameters of nanomaterials that interact with the immune system is also given in the second chapter of the "Handbook of Immunological Properties of Engineered Nanomaterials". 5

Quantification of the targeting moiety is another important nanomaterial property that needs to be addressed carefully before conducting in vitro and in vivo studies. Despite its importance for biological interpretation, there is not as yet much information on this topic. Attempts have been made to optimize ligand homogeneity as the surfaces of nanomaterials become increasingly complex in order to be used for biomedical applications. Many conventional chemical methods, such as nuclear magnetic resonance (NMR), absorption, and fluorescence spectroscopy, along with various chromatographic methods are useful in quantifying or at least confirming the presence of certain chemical groups on or in the surface of a nanomaterial, but they are unable to discern chemical differences between different loci on multiphasic nanomaterials. Other space-resolving characterization methods (such as energy dispersive X-ray spectroscopy) do not have the resolution to distinguish between small ligands, such as carboxyls, maleimides, and amines, which are commonly employed as reactive functionalities for future cross-linking reactions. ${ }^{8}$

The distribution of targeting moieties on the surface of a carrier is as relevant as their quantification because this will partially determine the final surface chemistry of the nanocarrier, which plays a key role in the carrier's biodistribution, pharmacokinetics, and toxicity. Patchy polymeric particles are clear examples of the unique distribution of targeting moieties that can be observed on the carrier surface..$^{9-11}$ For these reasons, it is important to characterize the three-dimensional distribution of target moieties. Few endeavors have been made in this regard due to the lower resolution available when using the more standard physicochemical techniques. Heterogeneity on a nanomaterial surface can potentially impact its performance in vivo, which makes it increasingly important to improve the experimental and analytical methods in this field, for which there is not an abundance of information. ${ }^{12}$ Other methods exist to quantify target moieties for particular types or aspects of nanomaterials in more general terms. For example, thermogravimetric analysis (TGA) is often used to quantify the thermal degradation of moieties. TGA lacks the 
ability to discern the spatial arrangement of these moieties in comparison with other methods, but it is a well established technique that is fairly straightforward and reproducible. ${ }^{13}$ TGA is limited by the degradation characteristics of the nanomaterial; easily degradable materials, such as biodegradable polymers, will be more difficult to quantify using TGA than inorganic nanomaterials because they contain common TGA leaving groups such as carboxyls. Hybrid electron microscopy techniques, such as scanning electron microscopy (SEM)Raman and energy-filtered TEM, discussed in the following section, may be employed to overcome some of the limitations of TGA and yield three-dimensional spatial details with sufficient sample processing time. ${ }^{14,15}$ In order to reconcile spatial selectivity with higher throughput, we recommend a combination of standard spectroscopic and chromatographic techniques and more modern nanometrology techniques to characterize nanocarriers more fully.

Ultraviolet-visible spectroscopy and fluorescence spectroscopy are fairly standard, low-cost, straightforward instrumental techniques for chemical analysis. Some nanomaterials have optical characteristics that hinder their analysis with ultraviolet-visible or fluorescence spectroscopy. Certain materials are transparent or refractive in the ultravioletvisible region and require additional functionalization in order for their moieties to be quantified via ultraviolet-visible or fluorescence spectroscopy. ${ }^{16}$ For example, Janus particles can induce refractions that distort the analysis of adjacent particles. ${ }^{16}$ In other experiments, only the fluorescentlylabeled part of the particle is excited during analysis. ${ }^{17}$ This process has often been accomplished via conjugation of fluorescent moieties (such as the Alexa Fluor line of fluorescent probes) to functional groups. ${ }^{9,12,18-20}$ When using this method, it is important to carefully select the fluorophores or chromatophores to avoid overlapping fluorescent wavelengths. Correct scientific interpretation will depend on the correct technical performance of the experiment.

Fourier transform infrared (FT-IR) spectroscopy is particularly useful for identifying the chemical nature (ie, the chemical "fingerprint") of a specific moiety present in the surface of the nanomaterial. FT-IR can also be used as a titration technique to detect relative amounts of different functionalities, but generally is not a quantitative tool. ${ }^{13,21}$ Attenuated total reflectance is a direct sample analysis technique that has been used for FT-IR analysis, and is particularly helpful for analyzing the chemical fingerprint of a nanomaterial without excessive sample preparation. Samples that are sensitive to the solvents or pressure required to prepare a typical FT-IR sample have been analyzed with this technique because
FT-IR spectra can be obtained from pure bulk materials on the attenuated total reflectance probe. ${ }^{22-25} \mathrm{NMR}$, especially solid state NMR, could potentially also be used to quantify amounts and locations of specific functional groups. NMR is more specific than FT-IR in that it can be used to target select nuclei. Hydrogen, carbon, and phosphorus compounds are commonly and routinely analyzed with NMR (as well as other nuclei, depending on their spin state and availability). ${ }^{12,20,26-28}$ NMR is also more qualitative than quantitative; it provides the chemical environment of the nucleus under analysis, and is not frequently employed (unless in titration) to quantify target moieties.

A number of elemental analytical techniques, including mass spectrometry and atomic emission spectrometry, are particularly advantageous for quantifying the physicochemical characteristics of nanomaterials. For example, matrix-assisted laser desorption ionization time-of-flight mass spectrometry has been used to quantify the size of inorganic nanoparticles, and has also been used to quantify organic polymeric nanomaterials. ${ }^{29,30}$ Inorganic particles have been ionized with inductively-coupled plasma (ICP) down to particle-scale resolution for analysis with ICP-mass spectrometry or ICP-atomic emission spectroscopy. ${ }^{31}$

Various liquid chromatography techniques can also be helpful for quantifying the targeting moieties on the nanoparticle's surface because they allow us to distinguish the heterogeneous ligand distribution in nanomaterial systems. Surface distribution of target moieties is directly related to the solubility behavior of the particles, and therefore chemical separations based on size and suspensibility are particularly useful for determining target moiety quantities. ${ }^{12}$ Related to these properties, $\mathrm{pH}$ and electrochemical titrations have also been employed to determine the binding efficiency of targeting moieties, but unlike chromatographic separation, titrations cannot be used to distinguish the effects of the three-dimensional arrangement of moieties. ${ }^{5,32}$ These electrochemical and chromatographic techniques are particularly useful for documenting and simulating the effects of chemical moieties on the surface of the nanomaterial if direct visualization is not possible.

With SEM hybrid techniques, nanomaterials can be directly imaged, and a targeting moiety present on the nanomaterial's surface can be specifically selected to obtain elemental information. An example of these SEM hybrid techniques is energy dispersive X-ray spectroscopy. Although this technique is highly effective in revealing the elemental composition of the target moieties, the resolution 
of the X-ray beam can span several microns, and therefore it is not the ideal instrument for identifying target moieties that are present on the nanomaterial's surface in less than 0.5 weight percent. In spite of their limitations, these standard characterization techniques are widely available and well understood. Still, as the surfaces of nanomaterials become more complex, the need to perform nanomaterial characterization with more powerful instruments and hybrid techniques increases.

\section{Recent developments in characterization techniques}

Until now the characterization of nanomaterials, particularly in the case of monophasic multifunctional polymeric nanoparticles, has been limited to measuring the size and surface charge of the nanocarrier. However, due to the diversity of numerous novel nanomaterials with intended uses in medical settings, metrology must be customized in order to characterize new nanomaterials appropriately. There are other limitations in the way we are presently characterizing nanomaterials. For example, the surface chemistry analysis of a nanocarrier that will be administered intravenously is poorly addressed. Also, the interplay between nanomaterial structure and function is often overlooked in the study of effects and toxicology of nanoscale structures. We believe that size, morphology (ie, external and internal), and surface chemistry are vital parameters that must be included in the characterization of a nanocarrier.

New developments in electron microscopy, materials science, and chemistry have provided several surface analysis techniques that can be very helpful in obtaining three-dimensional information about the surface and internal structure of a nanocarrier. A detailed surface and internal analysis of a nanocarrier will be extremely useful for achieving better control of the interaction between the nanocarrier, drug, and immune system. A wide range of microscopy instruments and techniques are available for this purpose, and include atomic force microscopy (AFM) with automated ultramicrotomy, scanning tunneling microscopy (STM), environmental SEM, electron loss microscopy, Z-contrast TEM, and low voltage electron microscopy. ${ }^{8,33}$

AFM provides "z-directional" resolution, mapping the topography of a material, and is a nondestructive technique that allows for further physicochemical characterization methods to be performed on the sample. AFM operates by measuring the atomic force of a cantilever probe impinging on an atomic surface, and the resulting signal is interpreted as the depth profile of the material, with resolution as low as the atomic scale. ${ }^{8,34}$ In-process control of particle formation, size measurements, stability, release, and degradation characteristics, as well as surface modifications can all be conducted via atomic force. ${ }^{35}$ Such precise detail on the nanomaterial surface is key to understanding the potential physical interactions between the surface of the drug delivery system (DDS) and the surrounding environment. Very few other methods are available that can resolve such small details for such a wide variety of sample types. In fact, AFM has been labeled as "nanoscopy" rather than microscopy with regard to emphasizing the level of detail achievable using this instrument. ${ }^{8}$

Automated ultramicrotomy and focused ion beam (FIB) are two new cross-sectioning techniques that are very useful for obtaining thin and reproducible slices of a particle's core. These two techniques are particularly relevant to illustrate the internal structure of polymeric nanoparticles. Automated ultramicrotomy is preferred for samples that could be damaged by the ion beam, but it is not a standardized, widely commercially available technique. Cross-sections of a set thickness are automatically cut and analyzed sequentially with AFM. ${ }^{33}$ In contrast, FIB allows for "real-time" slicing to take place. FIB cross-sections can be targeted at a particular location or for a particular depth for the nanomaterial. ${ }^{33}$ Of the many papers in the drug delivery field that report the use of conventional and new nanocarriers to deliver a specific cargo to the targeted organ, only a few papers report the internal structure of these nanocarriers. ${ }^{36,37}$ The development of robust nanocarriers for drug delivery purposes will require detailed internal characterization of these new carriers. It is essential to know whether the polymeric core is solid or hollow because the function of the particle's core depends on both internal and external physicochemical features. For example, internal structures might be formed within the polymeric core that can potentially interact with the payload and therefore influence the kinetics of drug release. In addition, characterization of the internal structure of polymeric particles is important because each chemical method used to synthesize polymeric particles might render particles with different internal structures. The drug delivery abilities of the nanocarrier are key for its therapeutic performance.

If automated ultramicrotomy or FIB are not feasible or available, TEM tomography is another powerful technique that can be employed to give internal and surface three-dimensional information about the nanomaterial. TEM typically gives a two-dimensional projection of a 
material's three-dimensional volume, and effectively serves as a single cross-sectional view of that material. Several different methods exist to generate cross-sectional images of nanomaterials using different TEM modalities and computational analysis. One such method involves tilting the axis of the sample and acquiring multiple images and then reconstructing the orthogonal projections in the tilt series into a three-dimensional image. ${ }^{38}$ Another method involves energy-filtered TEM, in which the elemental composition and distribution along three dimensions can be acquired by combining two-dimensional, energy-filtered TEM images with tomographic approaches. With nanometer-scale resolution, distribution of moieties on a surface can be determined accurately, along with the morphological characteristics of the host nanomaterial. ${ }^{14}$ These surface aspects mediate the direct interaction between the nanomaterial and the biological system.

The STM is an instrument that renders information about the surface chemistry of the nanomaterial for electrically conductive samples, providing topographical information similar to that obtained from AFM. ${ }^{8}$ STM gives atomic-scale details in various media, and these images can be compiled over time to visualize chemical processes at the atomic level. The atomic "movies" produced by STM illustrate and animate shifting atomic impurities and defects at the material's surface. Atomic movement on the surface could then be compared and interpreted with respect to the interactions between the nanomaterial and the biological system. Further, STM can be used for direct manipulation of atoms and molecules, inducing a chemical reaction, or even building a molecule by lateral or horizontal manipulations of atoms via the tunneling force. ${ }^{8}$ STM yields the atomic structure of the nanomaterial over time, allowing for a detailed understanding of its behavior and interaction with other atoms and molecules.

X-ray methods can yield more three-dimensional structural information regarding the degree of crystallinity, electronic band structure, and film thickness for the studied materials. For example, X-ray photoelectron spectroscopy is used to determine the elemental composition and electrical properties of materials. X-ray photoelectron spectroscopy has also been enhanced to map the three-dimensional structure of a $10 \mathrm{~nm}$ area at the surface of a material. Although X-ray photoelectron spectroscopy provides much useful data about the materials in question, it has extremely low resolution compared with more modern methods, and therefore typically needs to be combined with other methods to be relevant in a surface chemical analysis. ${ }^{8}$
X-ray diffraction is another important X-ray method that is typically employed to determine crystal structure in three dimensions for inorganic compounds. Beyond the simple crystal lattice structure of a material (or lack thereof in amorphous compounds), X-ray diffraction can also be utilized to map electron density on its surface. ${ }^{39}$ Nanoscale materials have distinct crystallinity compared with their larger-scale analogs, which gives them unique mechanical, optical, and electrical characteristics that will influence their interactions in the human body. ${ }^{39} \mathrm{X}$-ray diffraction distinguishes between amorphous phases as well, and can distinguish the crystal structure of proteins and other biological macromolecules, revealing the specific interactions that take place at the nanomaterial-biological interface..$^{27,34,39}$

The X-ray diffraction phenomenon can be manipulated to generate further details about a sample. For example, SAXS and wide angle X-ray scattering (WAXS) are synchrotron radiation-based techniques that give different structural data. SAXS can be used to analyze powders, films, or suspensions, while WAXS can yield other structural information about nanomaterials. ${ }^{8,40}$ Grazing interface-SAXS is employed for analyzing d-spacing of thin films. ${ }^{40,41}$ These techniques may also give information about porosity and accessibility of nanomaterials by determination of correlation lengths and radii of gyration. ${ }^{6}$ SAXS and WAXS are particularly useful for characterizing the surface of lipid-polymeric particles because many monophasic and hybrid nanoparticles are synthesized with lipids or lipid-PEGylated functional groups (LPFGs). The distribution and position of such lipids on the particle's surface is rarely mentioned in publications that use these particles as DDS. A detailed surface analysis of such types of particles is critical to ensure the delivery performance of these nanocarriers.

Raman spectroscopy can be used to monitor processes within nanomaterials over time (in solution, for example). Raman spectroscopy measures the inelastic scattering of a light source by a compound that occurs as a result of lowenergy, symmetrical vibrational and rotational modes of the compound, and can be performed on aqueous samples as an alternative to FT-IR. ${ }^{42,43}$ In contrast with electron microscopy, Raman spectroscopy relies on light and is not limited by the environmental conditions of an electron beam. This technique is typically used to analyze small-molecule moieties, but at the nanoscale, phonons induced by the incident optical field are confined by the spatial dimensions of the particle being analyzed, yielding unique Raman spectra for nanomaterials attributed to their size, allowing this technique to be applied for both molecular characterization and 
full-particle characterization. ${ }^{42,44,45}$ Raman spectroscopy, in combination with microscopy, can prove particularly useful for characterization of nanomaterials that have heterogeneous surfaces. ${ }^{46-50}$ WITec, a German company, has recently launched the first commercially available SEM-Raman instrument. $^{15}$

The general assumption with conventional Raman spectroscopy is that first-order scattering occurs at the "Brillouin zone", where the wave vector is zero. In essence, energetic waves are allowed to propagate freely in a particular direction because the phonon loses momentum as it traverses the dimensions of the material. At the nanoscale, however, backscattering occurs at the edge of the material (changing the phonon wave vector) such that uncertainty is induced into the measurement. ${ }^{44,45}$ Size, morphological symmetry, and other physical attributes of a particular nanosystem thereby induce calculable differences in the Raman spectrum. ${ }^{44}$ Raman spectroscopy can also be a very powerful technique to detect surface boundary characteristics for composite materials, which is important because nanomaterials have a very high ratio of surface area to particle mass. Considering the role the crystal lattice plays in propagating the phonons upon electromagnetic excitation, Raman scattering is analogous to X-ray diffraction in its relationship to the crystal structure, and can be used to characterize the relative amounts of crystalline and amorphous phase in the compound. ${ }^{44}$

Of the innovative characterization techniques described above, it is no accident that many of these methods combine two or more well-known methodologies. Certainly, an innovative researcher could develop a cornucopia of more modern techniques to meet the challenge of characterizing increasingly complex nanomaterials. Once a full understanding of the chemistry behind a nanostructure has been achieved, the logical next step is to determine the fate of the nanocarrier in vivo.

\section{Immunocompatibility of polymeric particles$$
\text { PEG chain density }
$$

Poly(ethylene glycol) (PEG) is a hydrophilic and flexible polymer that has been extensively used in the drug delivery field to provide the following nanocarrier properties: good suspensibility, a prolonged circulation half-life, an improved biodistribution profile, and an ability to bypass activation of the complement system. Although there are other polymers that can be used for these purposes, including dextran, PEG has been commonly used to coat a number of types of nanocarriers, including liposomes, ${ }^{51-53}$ polymeric nanoparticles, ${ }^{54}$ metallic organic particles, ${ }^{55-57}$ micelles, ${ }^{58-63}$ and carbon nanotubes. ${ }^{64-68}$ The preferential use of PEG for the synthesis of DDS over other polymers is mainly due to the fact that it can be further functionalized with both organic and inorganic moieties. In particular, functionalization of PEG with organic molecules such as enzymes, antibodies, and proteins is greatly advantageous because PEG does not markedly decrease the biological activity of these biomolecules. ${ }^{69}$ Incorporation of PEG on the surface of the particle can diminish activation of the complement system by preventing protein adsorption. ${ }^{70}$ Therefore, PEG plays a key role in determining the surface chemistry of the nanocarrier. Although PEG has been extensively used in the drug delivery field for the aforementioned reasons, the polymer chemistry aspect of PEG has not been fully investigated as yet. New knowledge on this aspect of PEG can help us to expand its functions as a key component in a DDS. It is well documented that PEG in aqueous solution displays two different configurations, ie, the mushroom and brush configurations. ${ }^{71}$ Depending on the PEG configuration and molecular weight, protein adsorption can be greatly diminished. However, to date, there is no rule of thumb about which configuration is the most suitable for a DDS. Selection of the PEG configuration for a DDS will mainly depend on its intended medical use.

We believe that investigating the polymer chemistry of PEG in depth could help us to design superior DDS. Contrasting small-molecule compounds, PEG and other polymers do not have a homogeneous molecular weight. Polymer molecules can be assumed to have a degree of polydispersity in molar mass. The stated molecular weight for synthetic polymers is simply an average of the molecular weights present in a polymer sample, and chemists may define this in terms of number-average, weight-average, viscosityaverage, or z-average. ${ }^{4}$ The polydispersity index, which is equal to the weight-average molecular weight divided by the number-average molecular weight, will vary depending on the purity of the starting compounds, the effectiveness of the catalysts employed, and the method and mechanisms of polymerization. There is some debate as to the most effective method to quantify polydispersity. Multiple methods exist, including gel permeation chromatography, DLS, and viscometry, each of which present unique benefits and limitations regarding reproducibility and accuracy. ${ }^{4}$ Manufacturers may or may not report the polydispersity of their samples, and in our experience they differ on what type of molecular weight range they report for their products. This is not a major concern with macroscale polymer applications for which the bulk properties of the polymers in question are 
more significant, but in the nanoscale drug delivery field, a difference of a few monomer units could potentially have a dramatic effect on drug binding and dispersal efficacy. For example, knowing that PEG with high molecular weight prevents protein adsorption more effectively than one with low molecular weight, because PEG with high molecular weight binds more water molecules, it allows for more rational DDS design, rather than trial and error. Differential scanning calorimetry thermographs show that PEG with different molecular weights have different glass transition temperatures, which reflect different thermodynamic behavior, and several distinct molecular weights. ${ }^{72}$ The desired properties of the particular PEGylated molecule could be affected by this unknown and unregulated polydispersity, so this polymeric property must not be overlooked in the development process for a DDS. ${ }^{26,73}$

\section{Conformation of PEG}

The orientation of PEG in aqueous solution will depend on the physicochemical properties of the molecule to which it is attached. PEG is synthesized with two different structures, ie, linear and branched. PEG that has a branched structure absorbs more water molecules than PEG with a linear configuration as shown by differential scanning calorimetry studies. ${ }^{69}$

Researchers have also found that the molecular weight of PEG has a strong influence on its thermodynamic behavior. PEG is a semicrystalline polymer and has a melting point below $67^{\circ} \mathrm{C}$, proportional to its molecular weight. ${ }^{74} \mathrm{PEG}$ also exhibits a glass transition temperature at which monomers begin to show segmental motion, and therefore is related to the chain length of each PEG molecule. Since PEGs of low molecular weight can be extremely polydispersed, they have multiple glass transitions in their differential scanning calorimetry thermograms at a wide range of temperatures. The presence of multiple $T_{g}$ values is a sign of polydispersity. ${ }^{72}$ The thermodynamic behavior of PEG is likely to influence the thermodynamic behavior of the entire polymer blend when PEG is one of the main polymers in the mixture, because PEG composites often feature direct surface-to-surface contact between different polymer groups.

A clear example of this phenomenon is the behavior of LPFGs. Such molecules are formed by a phospholipidpolymer conjugate (DSPE-PEG) and an end-terminal functional group (R), and are commonly used in the synthesis of different DDSs for several reasons. ${ }^{54}$ LPFGs allow us to functionalize PEG with a wide range of inorganic and organic molecules through the reactivity of the end-terminal functional group. Various functional groups are commercially available, enabling a number of different reactions and target moieties to be utilized. ${ }^{75}$ Just as polymer-solvent interactions determine the solubility of a polymer in a given solvent, polymer-polymer interactions between the various monomer units of different polymers can induce a certain degree of mixing (or lack thereof) which can be harnessed for biomedical applications. ${ }^{76}$ Characterization of PEG and DSPE-PEG-R by gel permeation chromatography is essential before synthesis of a DDS. Gel permeation chromatography is a technique that provides the number-average molecular weight and weight-average molecular weight. The degree of polymerization and polydispersity can vary from batch to batch. ${ }^{4}$ Commercial sources that manufacture and sell LPFGs provide neither molecular weight nor polydispersity data, and simply test for the presence of certain functional groups.

The solvent used for the synthesis and manipulation of the polymer also affects the chemical properties of PEG. The solubility of polymers is commonly estimated using either Hildebrand's or Hansen's solubility parameters. These estimations reveal how well a monomer interacts with a solvent or another monomer based on the principle of "like dissolves like". Depending on dispersion forces, the inherent polarization of the bonds of the molecule, and hydrogen bonding, a particular solvent may dissolve the compound more preferentially. ${ }^{76}$ This can directly affect the behavior of the DDS, in that a "good" solvent, for which the solubility parameters of the monomers and the solvent are closely matched, will yield a perturbed polymer for which the solvent molecules are distributed evenly around the entire polymer chain. A "bad" solvent will not perturb the polymer chains, and will be distributed around a tightly-coiled, self-interacting polymer. ${ }^{77}$ In DDS applications, for which nanocarriers are often suspended in buffers containing various salt concentrations, the nanocarrier-solvent interactions may vary. It is easily conceivable that the various cations and anions present in common buffers, such as phosphatebuffered saline or 2-(N-morpholino)ethanesulfonic acid (MES), may also alter the solubility properties of the nanocarriers; if nothing else, limiting the amount of water molecules that are available to surround the PEG. Perturbed and unperturbed PEGs could each have potential benefits for particular medical applications, and a transition from one state to another when the drug is administered intravenously would allow for kinetic-based applications.

LPFGs have been used extensively in the synthesis of polymeric particles. Some recent examples include pH-sensitive poly(histidine)-PEG/DSPE-PEG copolymer 
micelles for cytosolic drug delivery, ${ }^{28}$ poly(lactic-co-glycolic acid) (PLGA)-lecithin-PEG core-shell nanoparticles for cancer-targeted delivery, ${ }^{78}$ lipid-polymer hybrid particles, ${ }^{79}$ folate-targeted biodegradable polymersomes loaded with paclitaxel,$^{80}$ particles with multiple functionalized surface domains for controlled and sustained drug release, ${ }^{81}$ and fluorescent photoswitchable conjugated polymer nanoparticles. $^{82}$

Considering this abundance of research, it makes sense to discuss in depth the role of LPFGs in formation of the particles. Two years ago, our research group found that when particles were synthesized with PLGA and two different LPFGs, particles with segregated regions were observed. ${ }^{9}$ Each segregated region was formed by the presence of a different LPFG. This unexpected experimental finding indicates that the behavior of PEG can be influenced by molecules such as lipids and functional groups that are attached to it. Based on this experimental observation, it is important to pay attention to the behavior of PEG because PEG determines the surface chemistry of the DDS and ultimately its therapeutic performance. Particles with one or two surface domains render particles with different surface morphologies, which in turn can activate the complement system at a high, average, or low level. Further, it is important to study the arrangement of LPFGs in the patch when one, two, or more LPFGs are involved in particle synthesis. Small angle neutron scattering is a technique that might be useful to elucidate the arrangement of LPFGs in the patch. ${ }^{83}$ The charge of R and DSPE could potentially be stronger than the PEG contribution when solubility parameters are taken into consideration, in spite of the fact that PEG in the LPFG molecule occupies the highest volume in comparison with the DSPE fragment and the $\mathrm{R}$ terminal. As a result, the charge contribution of the end-terminal and the attached molecules might lead to movement of the whole molecule and determine its final arrangement. In addition, the interaction of the end-terminal functional groups and the solvent system is another important parameter that must be considered in particle synthesis. Such interactions might also play a key role in the final morphology of the particle. Understanding the behavior of PEG and PEG- $\mathrm{R}$ is essential to understand the protein-corona interaction that takes place in a DDS.

\section{Protein adsorption}

Protein adsorption is a very important topic that must be considered when we are designing a polymeric DDS. Understanding the protein adsorption phenomenon in depth will help us to accelerate the translational process. As mentioned before, PEG is the polymer most commonly used to prevent protein adsorption. The density and configuration of PEG have been considered to be parameters that prevent protein adsorption. There are some hypotheses stating that when PEG has a low molecular weight, only tightly-bound water is associated with the PEG chain. ${ }^{72}$ However, the mechanism by which PEG prevents protein adsorption is not completely understood.

As its molecular weight increases, the PEG chain begins to fold in on itself, forming segment-segment interactions as it traps additional, more loosely bound water between the segments. This water functions to bind together the polymer coil, making it difficult for proteins to interact with it. Thus, chain coiling and water hydration interactions provide the common link connecting a number of molecular weightdependent properties of PEG.

Several theories have been proposed to explain the low protein affinity of PEG both in surfaces and in solution. ${ }^{72}$ These include rapid mobility of the hydrated PEG chains, the large excluded volume of the PEG molecules, a repulsive force that results from a loss of configurational entropy when a protein approaches a PEG molecule, and the low interfacial free energy of the PEG-water interface and lack of protein binding sites (ionic and hydrophobic) on PEG. Many of these theories may be related to the unique properties of PEG molecules in aqueous solution. PEG is soluble in water at room temperature for a wide range of molecular weights, while related polymers such as poly(methylene oxide) and poly(propylene oxide) are water-insoluble at most molecular weights. Studies of PEG in aqueous systems suggest that a minimum of two and often three hydrogen-bonded water molecules are needed to satisfy the basic hydration of each ether unit of PEG. ${ }^{72}$

Studies on the kinetics of protein adsorption allow us to obtain better insights into the protein adsorption phenomenon..$^{32}$ Protein adsorption onto polymers results in a hard corona effect due to the polymer-protein chain intermingling that is effectively permanent. Subsequent layers of protein corona may form, but ultimately there are two kinetic rates involved in protein adsorption when permanent coronae are present, ie, formation of the first layer and formation of subsequent layers. Proteins in subsequent layers are relatively free to attach and detach from the corona. ${ }^{32,84}$ However, the complement protein $\mathrm{C} 3$ was found to bind covalently to other serum proteins only after they had formed a primary adsorption layer ${ }^{85,86}$ It was not established whether complement acts as a permanent corona, but certainly proteins that are not covalently bound do not have this behavior. For 
nonpolymeric nanoparticles in which the molecular unit is not chain-like, chain intermingling does not occur, so the initial protein corona and associated kinetics are not present. ${ }^{32}$

Continued scientific investigation of the protein adsorption phenomenon has recently revealed more intricate details. ${ }^{84}$ Milani et al reported their experimental observation of the formation of two types of coronas on the particle core. One is a "hard" corona, which is irreversible, and the other one is a "soft" corona, which is reversible. Using fluorescence correlation spectroscopy, transferrin-binding kinetics were found to contain two distinct steps. First, the hard corona forms, which is irreversibly bound to the particles. This is followed by the formation of a soft corona, which binds reversibly to the hard corona, forming a second layer. The time scale of protein adsorption and desorption for the soft corona was a number of minutes, whereas desorption of the hard corona took longer than the several hours in which the authors performed their analysis. This clearly delineates the corona formation phenomenon as two distinct steps. For soft corona formation, the kinetics of third-level, fourth-level, and higher-level corona formation appear to be in the same general time scale as second-level corona formation. ${ }^{84}$

Irreversible binding was found to act as a "memory function" in the nanoparticle. The specific proteins that form the primary coronal layer depend heavily on the physicochemical characteristics of the nanoparticle, and the binding of subsequent layers is dependent on the physicochemical characteristics of the first layer, which forms binding pockets with affinities for particular biological interactions. ${ }^{84}$ Therefore, it is possible that these in vitro and in silico protein adsorption studies could be used to predict the behavior of the nanomaterial once it becomes a biomaterial complex. This irreversible binding, and the kinetics associated with it, implies a conformational change in proteins exposed to the nanoparticles, which the authors describe as a "fingerprint" or "memory function". This presents a unique and interesting perspective regarding these particles, ie, the inner corona effectively has a memory storage function on a biochemical level, which indicates the specific order of binding of proteins to the surface. The authors note that studying the memory function phenomenon may prove very useful in nanomedical drug research because it indicates protein-drug interactions. $^{84}$

Another interesting recent discovery regarding protein adsorption was reported by Zeng et al. ${ }^{87}$ Using computational approaches and in vitro studies, the authors concluded that even with small changes in the surface chemistry of the materials, a significant change in protein binding affinity and protein-nanomaterial interactions was possible. Their computational studies revealed a binding pocket phenomenon in which small-scale domains of the head group of the capping material fit structurally into binding pockets of the protein to influence the interactions. The authors suggested using these methods to determine the interaction between nanomaterials and biological systems in drug discovery, because they allow for rapid assessment of particle surface properties, even if their elemental constituents are effectively the same. ${ }^{87}$

There has been much debate in the scientific literature regarding the occurrence and significance of the "Vroman effect", which postulates that proteins at lower concentrations in serum replace proteins at higher concentrations over time. ${ }^{7,84,88,89}$ If this effect is indeed present with nanomaterials, it seems that the rate of desorption and adsorption is very fast, and beyond the detection limit of many of the kinetic experiments that have been performed. Newer nanomaterials with complex morphologies and physicochemical characteristics are rapidly being synthesized, so it is more relevant to consider the nanocarrier-protein interactions than to confirm the extent to which the Vroman effect is taking place. With the increase in knowledge about proteomics in recent years, the literature describing and attempting to prove or disprove the Vroman effect has largely been replaced by more practical kinetic studies of protein adsorption. ${ }^{32}$ The physicochemical properties of the protein molecules must be contemplated as well.

A wide range of serum proteins adsorb spontaneously and nonspecifically on the surface of nanoparticles. ${ }^{90}$ The proteins themselves have unique surface chemistry and charge density that affect their adsorption behavior. For example, human serum albumin has the approximate geometry of a triangular prism with distinct positively charged patches that cause the protein to adsorb in a particular orientation. ${ }^{88}$ Succinylation or amination of lysine residues on human serum albumin, which alter the charge profile of this protein, significantly affected the adsorption behavior of these modified proteins on nanoparticles. ${ }^{88}$ Varying the $\mathrm{pH}$ or increasing the electrolyte concentration generally promotes coagulation of protein colloids, causing them to coalesce more favorably. ${ }^{91,92}$ These parameters must be taken into consideration when studying protein-nanoparticle interactions.

Surface-sensitive techniques, such as surface plasmon resonance (SPR) and quartz crystal microbalance with dissolution monitoring (QCM-D) have been utilized in recent years to study protein adsorption. Both of these techniques monitor the resonant frequencies of substrates on a surface. 
QCM-D detects the change in resonance frequency of a quartz crystal upon adsorption, whereas SPR detects changes in refractive index on adsorption sites on a chip. ${ }^{86,93}$ QCM-D has been used to detect protein adsorption on nanopatterned surfaces as well as to monitor complement adsorption onto the primary protein corona ${ }^{86,94}$ SPR can monitor the protein adsorption phenomenon with extreme precision, and has the ability to detect attomolar concentrations on a nanoparticle surface. ${ }^{93-95}$ SPR allows for monitoring of the kinetics of protein adsorption, allowing us to calculate binding rate constants directly and to examine the exact nature of the protein binding phenomenon. ${ }^{32}$ For example, SPR was coupled with isothermal titration calorimetry to quantify exchange rates and affinities of specific types of proteins on nanoparticle surfaces. ${ }^{32}$ Coupling these surface-sensitive techniques with more common electrophoretic techniques allows for clear, precise monitoring of protein adsorption on nanomaterials.

\section{New research approaches to drug delivery and particle synthesis that can potentially help to close the translational gap}

Current research approaches have been suggested to control the pharmacokinetic aspects of nanoparticles. For example, Moghimi et al suggest an integrated analysis approach that can help to reach clinical settings faster and more effectively. ${ }^{96}$ There is extensive research addressing important aspects of a DDS including characterization and pharmacokinetics. Nevertheless, independent studies in these areas without a connection between them will not help us to close the translational gap. The goal of this new research approach is to identify and establish the interdependency of size, shape, and surface characteristics of nanoparticles in relation to biodistribution, controlled drug release, and excretion. ${ }^{96}$ Within this umbrella research approach, three important aspects of DDS must be carefully addressed: the role of blood proteins in clearance kinetics; the role of geometric factors of DDS in relation to blood proteins; and bioavailability and repeated dosing. Each of these aspects is discussed briefly below.

A number of proteins are involved in the opsonization process in blood. The most prominent group of proteins in this process is the complement family, which have been found in a number of different studies to adsorb to nanomaterials that have not been properly coated with PEG to repel protein interactions. ${ }^{54,70,85,86,97}$ Although many proteins are actively involved in the clearance process in blood, if the nanocarrier has a neutral charge it might bypass activation of the complement system. ${ }^{89}$ Recently, it was found that denatured albumin, which forms a hard corona on nanomaterials, is directly involved in clearance of nanomaterials from the blood. ${ }^{98}$ Denatured albumin undergoes enhanced uptake by class A scavenger receptors on cells in the mononuclear phagocyte system, whereas native-state albumin does not interact with that system. ${ }^{98}$ Denaturation of this protein caused it to trigger an immune response that it would not normally elicit. If other proteins underwent similar structural changes upon adsorption to nanoparticles, they may also behave differently and cause different immune responses, different release kinetics, and different protein interactions than the naturally folded proteins.

The protein-particle interaction is certainly influenced by the geometry of the nanoparticle, including its overall three-dimensional shape as well as its surface topology. A number of studies have been done to determine the role of the nanocarrier surface and its internal geometry in the interaction between a DDS and blood proteins. ${ }^{99-101}$ For example, gold nanoparticles are often prepared as rods or spheres, and it was found that spheres $50 \mathrm{~nm}$ in diameter without PEG had higher uptake than the other particle types studied. The net surface charge of the particles also differed with variations in particle geometry: PEGylated gold nanorods had a greater net positive charge than spherical particles, and these differences affected particle uptake by prostate cancer cells. ${ }^{99}$ In another set of studies, surface roughness and porosity were modulated via variations in the voltage used in synthesis. ${ }^{91}$ The different surface topographies demonstrated different protein adsorption initially, but this was not found to have a profound effect on the biological fate of the nanoparticle. ${ }^{100,101}$ On the contrary, it was found that changes in the nanocarrier's shape (ie, sphere versus rod) have a pronounced effect on the fate of the nanocarrier. Thus, it seems that major variations in the shape of the DDS can have pronounced effects on the fate of the nanocarrier. Minor variations in carrier surface topography have a less pronounced effect.

Polymer chains, depending on their physicochemical properties, can be oriented in a variety of conformations at the surface of a DDS. It has been discussed repeatedly that PEG brush or mushroom configurations have a considerable effect on the surface chemistry of nanoparticles. Generally speaking, in the brush configuration, the density of PEG molecules must be very high in order to repel proteins, but using other polymers as patches in combination with hydrophilic 
polymers can also successfully reduce protein adsorption. ${ }^{102}$ The exact ratios of brush to patch must be tailored to optimize the protein-repelling effect, and consequently tailoring polymer geometry can yield specific effects and interactions with serum components. The size of the polymer brush can also greatly affect the formation of a protein corona, even with hydrophobic polymers. ${ }^{103}$ Certainly, the geometry of the nanoparticle must be taken into consideration when determining the bioactivity and bioavailability of these novel materials in medical applications.

Previous research has focused on reducing protein adsorption on different types of nanoparticles with dissimilar surface features. ${ }^{104-107}$ Various models exist to characterize the mechanism of formation of a protein corona. It is largely assumed that proteins bind to nonpolar surfaces because it is entropically favorable to do so. Surface adhesion of nonpolar proteins results from both an interaction between different nonpolar groups and destruction of the water clathrate that forms around the protein. ${ }^{7,106}$ Many peer-reviewed papers have discussed a change in the conformation of proteins as they undergo this adhesion process, which fundamentally affects their function in the blood. ${ }^{84,98,108}$ Two studies were conducted using five types of latex particles with different surface chemistries, and found that each different arrangement of ionic charges or hydroxyl groups on the surface of the particle induced different types of protein adsorption. ${ }^{105,107}$ These adsorption phenomena were compared using two-dimensional polyacrylamide gel electrophoresis to generate a protein pattern and compare it to the protein pattern of serum, which was not exposed to latex particles. The resulting protein pattern in the two-dimensional gel contained proteins similar to those found in the experiments using untreated serum, but at vastly different concentrations depending on the surface chemistry of the nanoparticles. ${ }^{109-112}$ The surface chemistry of the DDS plays a key role in the protein adsorption response, thereby mediating the pharmacological fate of the nanocarrier and its payload.

Nanocarriers have been shown to enhance the bioavailability of a number of payloads, achieving upwards of a 100-fold increase in bioavailability. ${ }^{113}$ A variety of administration routes can be utilized for nanomaterials, each of which present specific challenges depending on the physiological nature of the chosen mode of drug delivery. For example, in order for intravenously administered DDS to be biocompatible with blood, they must bypass the immune system to reach their target, so must repel proteins that could tag the DDS for removal. ${ }^{90,114}$ Attempts have been made to either functionalize the terminal ends of PEG or to add functional patches to PEGylated nanomaterials in order to make them biochemically reactive, with varying degrees of success. ${ }^{9,102}$ These considerations can be expanded to non-PEGylated nanocarriers, such as lipodot nanocarriers that were synthesized to image tumor cells; in contrast with PEGylated nanocarriers, lipidots were shown to be distributed in liver, adrenal glands, and ovaries rather than the heart, muscle, kidney, and brain. ${ }^{115}$

Nanomaterial DDSs are engineered to optimize the pharmacokinetics of a specific drug, allowing for more precisely targeted pharmaceutical treatments and less frequent repeat dosing. Reduction of injurious side effects of drug payloads is preferred. Liposomes and polymeric DDS, among the most well-established nanomaterials in wide use in the market, have been shown to have minimal deleterious effects after repeated dosing over several weeks. ${ }^{96}$ These systems can be used to enable sustained drug treatment without causing the complications associated with repeated dosing of their non-nanomaterial counterparts. For many DDSs, it has yet to be established whether the nanomaterial DDS produces quite the same physiological effect as its non-nanomaterial analog; indeed, the reduction in complications may be associated with a reduction in effective treatment. Engineered DDSs have been found to have novel toxicological effects as well, ranging from cardiotoxicity and neutropenia to palmar-plantar erythrodysesthesia. ${ }^{96}$ Between the benefits of enhanced bioavailability and reduction of complications associated with repeated dosing and the toxicological risks associated with DDS, a balance must be found in order to optimize DDS for translation. ${ }^{116}$

Standards for the dose concept of nanomaterials are largely absent in the scientific literature, which is likely a result of the intended specificity for medical applications of nanomaterials. ${ }^{117}$ Attempts have been made to characterize and regulate dosing in terms of residence time, surface area, and other biological interactions. ${ }^{117,118}$ Due to the interactions between nanomaterials and the human body and their influence on bioavailability as discussed above, appropriate dosing is not entirely straightforward for nanomaterials. Repeated dosing can be observed in vivo, in vitro, or ex vivo, with varying degrees of precision and reproducibility, in order to determine whether long-term exposure to a DDS may cause deleterious effects. ${ }^{119}$ Care must be taken to assess the dosing required to effectively elicit a biological reaction. ${ }^{120}$ The lack of standards in dose metrics makes it even more important for scientists to postulate and establish the biologically effective dose in order to accelerate the translation process. Dose metrics will vary depending on the disease state and treatment plan, and ignoring this aspect of the nanocarrier will render it useless for medical application. 
The biological fate along with the physicochemical characteristics of new nanocarriers must be ascertained through focused and directed study in order to translate nanomaterials from bench to bedside. Comprehensive characterization, in light of recent research on these topics, must include the most cutting-edge techniques in order to ensure an effective nanocarrier has been produced.

\section{Suggested standardized characterization techniques}

To summarize the most important characterization techniques to conduct a physicochemical analysis of a DDS as well as to carry out protein adsorption studies on the same DDS, we have selected a few important characterization steps that we believe are universally recommended for comprehensive characterization of a particular nanomedical system prior to bench to bedside translation. We distinguish between two prominent categories of nanomaterials: soft matter nanomaterials such as polymeric particles, and hard inorganic particles and carbon nanotubes. Certainly, nanomaterial study would benefit from as many characterization methods as possible, but we recommend a minimum of one method in each characterization category to be performed on each DDS. The order in which the characterization of the nanomaterial is performed is important as well: characterization techniques that involve the use of high radiation or high voltage to analyze the samples, such as electron microscopy, zeta potential, ICP, atomic emission spectrometry, mass spectrometry, and FIB, should be performed after the sample has been analyzed with nondestructive characterization techniques in order not to alter the physicochemical properties of the nanomaterial. The characterization techniques are summarized in Table 1. Once a thorough characterization of the nanomaterial is conducted, an integrated analysis of the protein interaction phenomenon must be performed. Qualitative, semiquantitative, and quantitative techniques for integrated analysis of protein interaction are summarized in Table 2. Using multiple techniques will help us achieve a certain threshold of understanding necessary for DDS translation.

\section{Regulatory issues on DDS}

At some point, a potential DDS that is successful in the laboratory research setting would and should make the translation to clinical testing and eventually to clinical use after a comprehensive understanding of its physicochemical characteristics and its interaction with the human body. This translation moves from the world of grants and publications to the world of drug development and regulatory submissions. In the section, we discuss in detail the FDA regulatory process through which the DDS needs to pass to be commercialized. The standardized characterization techniques included in Table 1 were deliberately chosen in order for potential FDA approval applicants to be ready to address the most important aspects of the FDA regulatory process, namely

Table I Suggested standardized characterization techniques to close the translation gap during the preclinical stage of the US Food and Drug Administration regulatory process (Part I)

\begin{tabular}{|c|c|c|c|c|c|}
\hline \multirow{2}{*}{\multicolumn{2}{|c|}{$\begin{array}{l}\text { Characterization } \\
\text { of particle's main } \\
\text { structural components }\end{array}$}} & \multicolumn{2}{|c|}{ Characterization techniques } & \multirow{2}{*}{$\begin{array}{l}\text { Physicochemical } \\
\text { information acquired } \\
\text { from the nanocarrier's } \\
\text { components }\end{array}$} & \multirow{2}{*}{$\begin{array}{l}\text { Biological or therapeutic } \\
\text { outcome }\end{array}$} \\
\hline & & $\begin{array}{l}\text { Soft matter } \\
\text { (polymeric particles) }\end{array}$ & $\begin{array}{l}\text { Inorganic particles } \\
\text { and carbon nanotubes }\end{array}$ & & \\
\hline \multicolumn{2}{|l|}{ Core } & $\begin{array}{l}\text { Automated } \\
\text { ultramicrotomy, TEM, FIB }\end{array}$ & TEM, FIB & $\begin{array}{l}\text { Nanocarrier's internal } \\
\text { structure and core } \\
\text { chemistry }\end{array}$ & $\begin{array}{l}\text { Better understanding } \\
\text { between drug and carrier } \\
\text { to improve biodistribution } \\
\text { and pharmacokinetics } \\
\text { of the DDS }\end{array}$ \\
\hline \multirow[t]{4}{*}{ Surface } & A. Structure & $\begin{array}{l}\text { SEM, AFM, SEM-Raman } \\
\text { confocal-Raman }\end{array}$ & SEM, STM, AFM & $\begin{array}{l}\text { Nanocarrier's external } \\
\text { structure and texture }\end{array}$ & $\begin{array}{l}\text { Improved understanding } \\
\text { of the targeting }\end{array}$ \\
\hline & B. Charge & DLS & DLS & Carrier's charge & ability, biodistribution, \\
\hline & C. Size & DLS, GPC, SAXS & $\begin{array}{l}\text { DLS, MALDI-TOF-MS, } \\
\text { ICP-MS }\end{array}$ & $\begin{array}{l}\text { Carrier's size } \\
\text { polydispersity }\end{array}$ & $\begin{array}{l}\text { pharmacokinetics, and } \\
\text { toxicity profile of the }\end{array}$ \\
\hline & $\begin{array}{l}\text { D. Quantification } \\
\text { of target moieties }\end{array}$ & $\begin{array}{l}\text { Fluorescent labeling, } \\
\text { TEM-tomography, HPLC }\end{array}$ & $\begin{array}{l}\text { Fluorescent labeling, } \\
\text { TGA, TEM-tomography, } \\
\text { EDS, HPLC, ICP-MS, } \\
\text { ICP-AES }\end{array}$ & $\begin{array}{l}\text { Quantity and spatial } \\
\text { distribution of targeting } \\
\text { moieties, heterogeneity } \\
\text { of ligands }\end{array}$ & $\begin{array}{l}\text { DDS and improved batch } \\
\text { reproducibility }\end{array}$ \\
\hline
\end{tabular}

Abbreviations: AFM, atomic force microscopy; DDS, drug delivery system; DLS, dynamic light scattering; GPC, gel permeation chromatography; HPLC, high-performance liquid chromatography; MALDI-TOF, matrix-assisted laser desorption ionization time-of-flight; MS, mass spectrometry; TGA, thermogravimetric analysis; AES, atomic emission spectroscopy; EDS, energy dispersive X-ray spectroscopy; FIB, focused ion beam; SAXS, small-angle X-ray scattering; ICP, inductively-coupled plasma; SEM, scanning electron microscopy; STM, scanning tunneling microscopy; TEM, transmission electron microscopy. 
Table 2 Analysis of protein interaction

\begin{tabular}{|c|c|c|c|}
\hline $\begin{array}{l}\text { Type of analysis of } \\
\text { protein interaction }\end{array}$ & Technique & Information acquired & Biological and therapeutic outcomes \\
\hline Qualitative & 2D PAGE & $\begin{array}{l}\text { Identification of types of proteins bound } \\
\text { to the nanocarrier }\end{array}$ & $\begin{array}{l}\text { Improved biodistribution, bioavailability, } \\
\text { blood compatibility, and pharmacokinetic }\end{array}$ \\
\hline Semiquantitative & MALDI-TOF-MS & $\begin{array}{l}\text { Mass and relative abundance of proteins } \\
\text { bound to the nanocarrier }\end{array}$ & aspects of DDS \\
\hline Quantitative & SPR & $\begin{array}{l}\text { Real-time monitoring of kinetic rate } \\
\text { constants of protein binding }\end{array}$ & \\
\hline
\end{tabular}

Abbreviations: 2D PAGE, two-dimensional polyacrylamide gel electrophoresis; DDS, drug delivery system; MALDI-TOF, matrix-assisted laser desorption ionization time-of-flight; MS, mass spectrometry; SPR, surface plasmon resonance.

the safety and efficacy of the evaluated product at any stage of the translation process.

\section{Overview of the IND process}

The process of developing a new drug or a DDS typically proceeds along a relatively predictable sequence. An overview of this process is shown in Figure 1 and shows the progression from bench to bedside. This translation process includes the preclinical (ie, in vitro and in vivo studies) and clinical (ie, clinical trials) development as well as the FDA regulatory and commercialization processes. The complete process can take 10-20 years and requires many hundreds of millions of dollars to get to product launch. ${ }^{121}$ There are two major regulatory checkpoints in this process, ie, the Investigational
New Drug (IND) submission and eventually the New Drug Application (NDA) submission. The IND is a request from a company or clinical investigator to the FDA for permission to begin human clinical trials with a new drug. The NDA is filed after all nonclinical and clinical studies are complete and is the formal request from the company for approval to begin marketing the new drug. ${ }^{122}$ For this discussion, we will provide a high-level overview of the process in getting from development to the point of first in human IND studies and connecting the FDA requisites with the standardized techniques included in Table 1. These procedures apply to any material used as a drug, ranging from the most sophisticated polyfunctional nanoparticle to the simplest minor molecular variant "me-too" small-molecule drug.

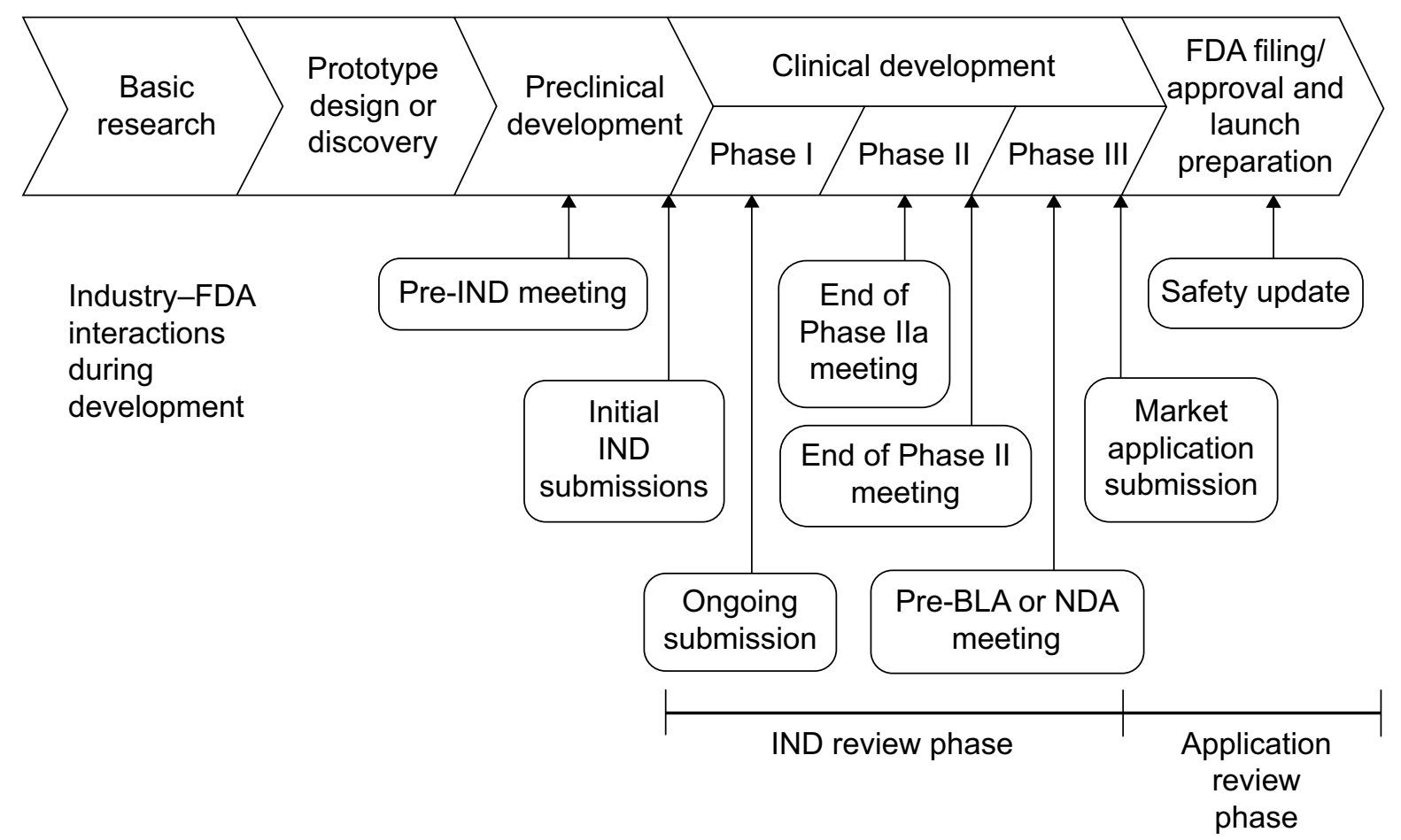

Figure I Drug development process.

Abbreviations: BLA, Biologics License Application; FDA, US Food and Drug Administration; NDA, New Drug Application; IND, Investigational New Drug. 


\section{Major guidance documents}

The FDA has published a large series of official documents that provide regulatory guidance on specific aspects of drug development. These are available on the FDA website (http:// www.fda.gov) along with a large amount of other information on drug development. The specific guidance documents can be accessed at http://www.fda.gov/Drugs/GuidanceComplianceRegulatoryInformation/Guidances/default.htm, and are subdivided into logically related categories. A subset of the guidance documents most likely to apply is shown in Table 3. There are many other guidances that could come into play depending on the specifics of the drug and of the disease that the drug is intended to treat. The FDA has not established regulatory definitions of "nanotechnology", "nanomaterial", "nanoscale," or other related terms. In 2014, the FDA issued a final guidance for industry reflecting the agency's current thinking about how to determine whether a product involves nanotechnology. This document outlines overarching considerations for all FDA-regulated products, including for drugs (Table 3 ). At this time, when considering whether an FDA-regulated product involves the application of nanotechnology, the FDA will ask if a material or end product is engineered to have at least one external dimension, or an internal or surface structure, in the nanoscale range (approximately 1-100 nm).

In addition, because materials or end products can also exhibit related properties or phenomena attributable to a dimension(s) outside the nanoscale range of approximately 1-100 $\mathrm{nm}$ that are relevant to evaluations of safety, effectiveness, performance, quality, public health impact, or regulatory status of products, FDA officials will also ask if a material or end product is engineered to exhibit properties or phenomena, including physical or chemical properties or biological effects, that are attributable to its dimension(s), even if these dimensions fall outside the nanoscale range, up to one micrometer $(1,000 \mathrm{~nm})$.

These considerations apply not only to new products, but also when changes to manufacturing processes alter the dimensions, properties, or effects of an FDA-regulated product or any of its constituent parts. Additional information has been published in a series of publications in the scientific literature, which are discussed below.

The IND submission is not a single document but rather a whole series of documents and laboratory study reports. A good overview of major parts of the drug development process can be found in the International Conference on Harmonisation (ICH) M3 guidance (Table 3). The IND components can be grouped into three major areas: pharmacology, toxicology and chemistry, and manufacturing. Depending on the specifics of the situation, there may be additional reports covering special areas such as immunotoxicity. Finally, the information is distilled into a specific document for use by clinical investigators who would (hopefully) be conducting the human clinical trials. Note that the pharmacology and toxicology studies for the IND are usually conducted in compliance with Good Laboratory Practice standards and that the production of the drug materials should be in compliance with current Good Manufacturing Practice (GMP) standards (Table 3). As mentioned above, it is important that nanomaterials fit to a certain degree of

Table 3 Major US Food and Drug Administration regulatory guidance documents

\begin{tabular}{|c|c|c|}
\hline Class & Title & URL \\
\hline General & $\begin{array}{l}\text { Considering whether an FDA-regulated product } \\
\text { involves the application of nanotechnology }\end{array}$ & $\begin{array}{l}\text { http://www.fda.gov/Regulatorylnformation/Guidances/ } \\
\underline{\text { ucm } 257698 . h t m}\end{array}$ \\
\hline Safety & $\begin{array}{l}\text { M3(R2) nonclinical safety studies for the conduct } \\
\text { of human clinical trials and marketing authorization } \\
\text { for pharmaceuticals }\end{array}$ & $\begin{array}{l}\text { http://www.fda.gov/downloads/Drugs/ } \\
\text { GuidanceComplianceRegulatorylnformation/Guidances/ } \\
\text { UCM073246.pdf }\end{array}$ \\
\hline Pharmacology/toxicology & $\begin{array}{l}\text { Content and format of investigational INDs for } \\
\text { Phase I studies of drugs, including well characterized, } \\
\text { therapeutic, biotechnology-derived products }\end{array}$ & $\begin{array}{l}\text { http://www.fda.gov/downloads/Drugs/ } \\
\text { GuidanceComplianceRegulatorylnformation/Guidances/ } \\
\text { UCM074980.pdf }\end{array}$ \\
\hline Pharmacology/toxicology & $\begin{array}{l}\text { Toxicokinetics: the assessment of systemic } \\
\text { exposure in toxicity studies (ICH S3A) }\end{array}$ & $\begin{array}{l}\text { http://www.fda.gov/downloads/Drugs/ } \\
\text { GuidanceComplianceRegulatorylnformation/Guidances/ } \\
\text { UCM074937.pdf }\end{array}$ \\
\hline Pharmacology/toxicology & $\begin{array}{l}\text { Pharmacokinetics: guidance for repeated } \\
\text { dose tissue distribution studies (ICH S3B) }\end{array}$ & $\begin{array}{l}\text { http://www.fda.gov/downloads/Drugs/Guidance } \\
\text { ComplianceRegulatorylnformation/Guidances/ } \\
\text { UCM074938.pdf }\end{array}$ \\
\hline Pharmacology/toxicology & $\begin{array}{l}\text { Immunotoxicity studies for human } \\
\text { pharmaceuticals (ICH S8) }\end{array}$ & $\begin{array}{l}\text { http://www.fda.gov/downloads/Drugs/ } \\
\text { GuidanceComplianceRegulatorylnformation/Guidances/ } \\
\text { UCM074965.pdf }\end{array}$ \\
\hline
\end{tabular}

Abbreviations: FDA, US Food and Drug Administration; IND, Investigational New Drug; ICH, International Conference on Harmonisation. 
homogeneity according to current GMP recommendations. According to the Guidance For Industry: Current GMP for Phase I Investigational Drugs, specified attributes should be monitored and acceptance criteria applied appropriately. ${ }^{123}$ Although there are no specific recommendations in place with regard to surface homogeneity, it is an important parameter to consider when developing more complex nanomaterial-containing DDS. The FDA guidance documents present several prerequisites that the applicant needs to meet to safely bring new medical innovations to the stage of human clinical trials. These prerequisites are often overlooked during the preclinical stage of the nanomaterial because of its limited characterization. The standardized techniques presented in Table 1 when properly utilized can help the applicant to better prepare to satisfy the FDA regulations at both the experimental and translation levels by providing a set of standardized techniques to test the performance of the DDS at any stage of its synthesis and during the manufacturing process.

\section{Pharmacology: absorption, distribution, metabolism, and excretion}

Studies in this section of an IND report the results of the nonclinical studies evaluating the pharmacology aspect of the new drug or DDS. These studies cover the absorption, distribution, metabolism, and excretion (ADME) of the drug or DDS, and are usually performed in two species, one rodent and one nonrodent. A single species can be used where the active pharmaceutical ingredient (API) is only active in one nonclinical species. This usually applies where the API is a therapeutic protein that is active in primates but not in rodents or canines (Table 3 ). The purpose of these studies is to provide a complete lifecycle view of the drug in vivo. This view includes each component of the nanomaterial-containing drug product. The analysis should include the actual API, the supporting particle, and any additional components, such as targeting ligands or protective groups. If the particle is degraded, then the analysis should include an evaluation of the subcomponents. This analysis is particularly relevant for applications that include soluble metal nanoparticles and biodegradable polymeric nanomaterials. ${ }^{124}$ This is analogous to the evaluation of small-molecule drug metabolism. If the particle is not degradable, then an evaluation of effects at any sites of accumulation would be needed.

One of the major problems that the FDA has identified in the ADME evaluation process is the limited information provided by the applicant about the detection of degradation products of the nanomaterial during or after its functional lifetime. Proper assessment of degradation products requires a careful and deep analysis of the nanomaterial after it has been in contact with the body. Table 1 lists a series of techniques that can help to reduce this problem. For example, SPR is a technique that allows us to conduct kinetic studies of the in vitro degradation processes of a DDS over time ${ }^{117}$ and to detect these degradation products in the order of micrograms or nanograms. ${ }^{125}$ Data collected from in vitro studies can guide the selection of methods to evaluate samples from in vivo pharmacology and toxicology studies. If the applicant conducts in vitro, ex vivo, and in vivo experiments monitoring DDS degradation products over time using the appropriate instrumentation, the risk of the DDS failing in clinical trials could be greatly reduced. In addition to SPR, matrix-assisted laser desorption ionization time-of-flight mass spectrometry, ICP-MS (mass spectrometry), and ICP-AES (atomic emission spectroscopy) can detect trace levels of degradation products for metallic nanoparticles. ${ }^{30,31,125}$ High-performance liquid chromatography and gel permeation chromatography separate compounds based on their physical characteristics (eg, solubility and size) and can distinguish between soft matter nanomaterials if their degradation products exhibit different physical characteristics in the chromatography column. ${ }^{20,87,126}$ In either case, a comprehensive assessment of the nanomaterial's degradation products derived from its exposure in vivo should be conducted. The degradation products might be due to physicochemical changes that may have occurred during the nanomaterial's functional lifetime. ${ }^{127}$ Protein adsorption is a phenomenon that often changes the physicochemical properties of a nanomaterial, and such interaction might have an effect on the medical performance of the nanomaterial. A more specific discussion of ADME studies as they relate to nano material-containing drugs has been published. ${ }^{128}$

\section{Toxicology}

Toxicity is one of the most important aspects of a product that the FDA regulatory process will evaluate. The primary screening methods are standard histopathology and clinical chemistry studies. Where toxicity is observed, it is helpful to be able to provide a mechanistic explanation for the observed toxicity. For some types of nanoparticles, toxicity may be caused by the nanomaterial's degradation products. One important way to assess this type of toxicity is based on the presence of biochemical markers of oxidative stress such as reactive oxygen species. ${ }^{129,130}$ Other "non-ROS-y" toxicological outcomes have also been documented with nanomaterials. ${ }^{130,131}$ Oxidative stress does not directly reveal the presence and physicochemical nature of relevant toxic compounds, such as degradation products and undesired 
DDS-protein interactions. Information developed in the course of ADME studies to identify degradation products can be very useful to guide the applicant in conducting a thorough characterization of the toxicity of the nanomaterial and any breakdown products in vitro and in vivo. In addition, a comprehensive study of the interaction between the DDS and proteins may also provide useful information on the observed toxicity. The toxicity of the DDS can be assessed at the protein, tissue, and cellular levels as needed to explain any observed toxicity. Recent developments in microscopy, analytical chemistry, optics, and spectroscopy, such as some of the hybrid analytical instruments indicated in Table 1, are highly useful for deep characterization of the DDS. Raman microscopy, including confocal Raman and SEM-Raman, is one of the most powerful techniques that exist today. It is a technique that is particularly relevant for toxicity studies because it does not require the use of fluorescent labels or staining procedures. ${ }^{47-50}$ Fluorescent labels can alter the surface chemistry of the DDS, and such alterations can mask signs of toxicity exclusively due to the nanomaterial or potentially create toxicity that might not otherwise exist. Confocal-Raman is an instrument from which a substantial amount of information on the surface chemistry of the nanomaterial can be obtained. Morphology, topology, and chemical composition can be determined for both metallic and soft matter nanoparticles with this instrument, revealing potential toxicity risks at a chemical level. ${ }^{47-50}$

Nonclinical toxicology studies are required for all new drugs or DDS. These include single-dose and repeat-dose study designs. The goals of these studies are to identify the maximum dose that does not cause any adverse effects and to identify any toxicity that is caused by the drug or DDS. In practice, data from these in vivo studies are commonly used to provide information for both ADME and toxicology reports. These studies should be designed to be as close to the intended clinical use as is practical. The specifics of the studies can be significantly affected by a number of factors. These include the details of the design of the drug or DDS, the disease that it is intended to treat, the expected duration of treatment, and the safety and efficacy of existing treatments for the disease. It is recommended to have regular consultation with the regulatory authorities to ensure that studies are properly designed and that all needed endpoints have been incorporated.

\section{Chemistry, Manufacturing, and Control}

The Chemistry, Manufacturing, and Control (CMC) section of the FDA application is very important in the case of nanomaterial-containing drugs. The composite nature of these drugs may result in significant additional complexities beyond those of small-molecule or protein drugs. The purpose of this section is to describe the sources and specifications of each material that is involved in the synthesis of the complete drug product. The CMC process would include quality evaluation of each component, a description of the production sequence, quality evaluations at intermediate steps, and a full evaluation of the finished nanomaterial-containing active drug product and of any excipients in the formulation. The standardized techniques mentioned in Table 1 are robust and sensitive enough to allow researchers to examine the physical stability, chemical stability, and purity of the key components of a DDS at each step of synthesis and in the manufacturing processes. Using these techniques, physicochemical changes produced in the DDS during its medical performance can be identified and measured. For example, AFM can detect three-dimensional topographical changes in nanomaterials both as solids and in suspensions, allowing for analysis of "dry" precursors as well as suspensions or solutions. ${ }^{34,35,87,103}$ The surface structures and textures of the DDS can be precisely imaged using this technique, revealing the stability of the sample over time. High-performance liquid chromatography is a powerful technology that can be especially important in the CMC process because it can separate compounds in the sample based on their solubility properties. Solubility is directly related to the surface chemistry of the nanomaterial. Hence, high-performance liquid chromatography can distinguish samples based on spatial distribution and heterogeneity of ligands. This can be used for monitoring chemical changes that occur during the manufacturing process $^{12}$ as well as evaluating the homogeneity of the DDS. In addition, that high-performance liquid chromatography instruments are commonly outfitted with autosamplers and quantitation software, so allow the conduct of a highthroughput automated analysis of numerous samples, which helps accelerate the translation process. Raman microscopy is another powerful technique that allows for robust process analysis. This instrument can help the applicant assess the strength, quality, and purity of the drug or DDS. Samples do not require staining or fixation and are not destroyed during the analysis process. Chemical features of the sample, including some three-dimensional conformation information, can be deduced as a result of molecular vibrations induced by the Raman laser. The presence of impurities, aggregates, or degradation products in the sample would be revealed by a characteristic Raman pattern exclusive 
from that of the original compound. ${ }^{8,42}$ In addition, optical phonon confinement in Raman spectroscopy can reveal the physical features of nanomaterials during the manufacturing process. ${ }^{44}$ In this way, this instrument can help the applicant monitor the chemical reactivity of the drug or DDS along the synthesis process. Also, one of the greatest advantages of this instrument for the chemical and manufacturing aspect of the drug is that the samples can be analyzed in the solid or liquid phase. As with AFM, Raman microscopy can be used with dry or wet samples, which are equally relevant for manufacturing processes. The data obtained during the $\mathrm{CMC}$ process assure the regulatory authorities that the sponsor has a full understanding of the production process and can produce multiple lots of the same product in compliance with appropriate quality and purity specifications. ${ }^{122}$ A small investment in early compliance with current GMP in sources and methods can pay off in not having to redo important pilot studies. This is an area where an understanding of quality by design principles and practices can be of real practical benefit, especially for scientists without manufacturing experience. Table 1 presents a set of characterization techniques that may be useful to conduct a comprehensive assessment of various aspects of quality of the DDS, overcoming existing challenges and helping close the translational gap. The guidance documents for the CMC area are more tightly focused than those in other areas. The specific guidance documents that apply will depend on the specific composition of the drug or DDS. If it includes a therapeutic protein as an API, then several guidance documents covering therapeutic protein manufacturing and analysis might come into action. A small-molecule drug as an API would bring other documents into play. It would be important to have early consultation with the regulatory authorities in order to have clarity on what documentation needs to be produced in compliance with which regulatory documents. Table 4 includes suggested standardized characterization techniques that can be useful to close the translation gap during the preclinical stage of the FDA regulatory process.

\section{Special guidances}

In addition to the general guidance documents, there are more specialized guidances that may apply again depending on the composition of the nanomaterial-containing drug. One area of common concern is that of immunotoxicity. There are two guidance documents that may apply. The first is the ICH S6 document, which covers issues related to the potential for immunotoxicity of therapeutic proteins. If a nanoscale particle uses a protein either as an API or as a specific targeting ligand, which is often the case, then this document would apply. The primary concerns are for immunogenicity of the proteins and how this might affect the pharmacological activity of the drug (Table 3 ). The other document that could apply is the ICH S8 document (Table 3). This covers more general areas of drug-associated modulation of the immune system. The clause of specific application for nanoparticle drugs is the one that covers accumulation of drugs in cells of the immune system. Nearly all nanoparticles are eventually cleared from the circulation or tissues by tissue macrophages, granulocytes, and Kupffer cells. As most particles are not rapidly biodegradable, they will accumulate, often to very high levels. ${ }^{132}$ The sponsor should consider how this accumulation might affect the cells where the particles accumulate.

\section{Clinical}

The final major area of an IND is the information that is more directly related to the planned clinical trials. As in the $\mathrm{CMC}$ area, there are a large number of specialized guidance documents. These are mostly focused on development of drugs for a specific disease or group of related clinical conditions.

Table 4 Suggested standardized characterization techniques to close the translation gap during the preclinical stage of US Food and Drug Administration regulatory process (Part II)

\begin{tabular}{|c|c|c|}
\hline $\begin{array}{l}\text { Key evaluation FDA regulatory } \\
\text { processes required in an IND } \\
\text { application }\end{array}$ & $\begin{array}{l}\text { Challenges faced during synthesis, manufacture, } \\
\text { and medical performance of a DDS in the } \\
\text { preclinical stage of the FDA approval process }\end{array}$ & $\begin{array}{l}\text { Useful characterization techniques } \\
\text { to address specific challenges } \\
\text { during FDA regulatory processes }\end{array}$ \\
\hline $\begin{array}{l}\text { Absorption, distribution, metabolism, } \\
\text { and excretion }\end{array}$ & $\begin{array}{l}\text { Degradation products, protein adsorption, and particle } \\
\text { accumulation }\end{array}$ & $\begin{array}{l}\text { SPR, MALDI-TOF-MS, ICP-MS, ICP-AES, } \\
\text { HPLC, GPC }\end{array}$ \\
\hline Toxicology & $\begin{array}{l}\text { Detection of signs and causes of toxicity at the protein, } \\
\text { cellular, and tissue levels }\end{array}$ & $\begin{array}{l}\text { Confocal-Raman, SEM-Raman, EDS, ROS } \\
\text { and non-ROS toxicity assays }\end{array}$ \\
\hline $\begin{array}{l}\text { Chemistry, Manufacturing, } \\
\text { and Control }\end{array}$ & $\begin{array}{l}\text { Purity, homogeneity, chemical stability, aggregation, and } \\
\text { degradation }\end{array}$ & AFM, Raman, HPLC \\
\hline
\end{tabular}


The documents apply to any type of drug intended to treat the covered condition, irrespective of whether it is a small molecule, therapeutic protein, or advanced multicomponent nanoparticle. Because nanotechnology is a highly interdisciplinary field, care must be taken to ensure that these trials are planned and performed by clinically experienced individuals. In this section, the sponsor will also lay out their proposed designs for the Phase I clinical studies in detail. These plans will include patient criteria, dosing schemes, and specifics of monitoring for toxicities, as well as full data collection and statistical analysis designs. They will also include contingency plans for responses to drug toxicity whether expected or not. The end goal is a full description of the planned human studies that carefully balance protecting the health of the study subjects with obtaining the data needed to proceed to the later stages of drug development (Table 3).

Successful translation from the research world to the regulated world of drug development requires careful attention to detail. This requires the ability and the funding to perform pre-clinical development and to generate the correct documentation. The end goal is to provide the regulatory authorities with sufficient data to demonstrate that the investigational drug is sufficiently safe and has plausible evidence of potential for clinical efficacy.

\section{Conclusion}

The time has come to bridge the translational gap between bench-top scientific research and bedside medical applications with nanomaterials, considering the great deal of time and financial resources that have been invested in development of nanotechnology. In recent years, the diversity and availability of complex nanomaterials has skyrocketed. Therefore, standardized techniques must be established to characterize these novel nanomaterials prior to translation. Fortunately, a number of powerful instruments in electron microscopy, spectroscopy, and analytical chemistry are commercially available to conduct thorough characterization of nanomaterials, which will allow us to acquire a deeper understanding of their physicochemical properties. Equally important, it is imperative that the scientific community expands its understanding on the interaction between the DDS and cells, tissues, and proteins in vivo and in real time, as biological interactions in physiological fluids are key for the practical use of nanoparticles in medicine. We have suggested a minimum universal set of characterization techniques that can be applied to new nanosystems. This list can be further developed and refined as the field evolves. A permanent and close collaboration between academia, industry, and the FDA is indispensable for accelerating the translation process of DDS.

\section{Acknowledgments}

This work was supported by the CSM (Carolina SalvadorMorales) startup fund (162904). AAK is supported by a George Mason University Graduate Research Assistantship from the CSM startup fund.

\section{Disclosure}

The findings and conclusions in this paper have not been formally disseminated by the US Food and Drug Administration and should not be considered to represent any agency determination or policy. The authors report no other conflicts of interest in this work.

\section{References}

1. Executive Office of the President of the United States. The National Nanotechnology Initiative Supplement to the President's 2015 Budget. Washington, DC, USA: National Science and Technology Council; 2014. Available from: http://www.whitehouse.gov/sites/default/files/ microsites/ostp/NNI_FY15_Final.pdf. Accessed October 3, 2014.

2. Roko MC, Mirkin CA, Hersam MC. WTEC panel report on nanotechnology research directions for societal needs in 2020: retrospective and outlook. Washington, DC, USA: World Technology Evaluation Center Inc; 2010 Available from: http://www.wtec.org/nano2/Nanotechnology_Research_ Directions_to_2020/. Accessed October 3, 2014.

3. Matsumura Y, Maeda H. A new concept for macromolecular therapeutics in cancer chemotherapy: mechanism of tumoritropic accumulation of proteins and the antitumor agent smancs. Cancer Res. 1986;46(12 Pt 1):6387-6392.

4. Rodriguez F, Cohen C, Ober CK, Archer LA. Molecular weight distribution and average molecular weight. Principles of Polymer Systems. 5th ed. New York, NY, USA: Taylor and Francis Books Inc; 2003.

5. Clogston J, Patri AK. Importance of physicochemical characterization prior to immunological studies. In: Dobrovolskaia MA, McNeil SE, editors. Handbook of Immunological Properties of Engineered Nanomaterials. Volume 1. Hackensack, NJ, USA: World Scientific Publishing; 2012.

6. Ehmann HM, Spirk S, Doliška A, et al. Generalized indirect fourier transformation as a valuable tool for the structural characterization of aqueous nanocrystalline cellulose suspensions by small angle x-ray scattering. Langmuir. 2013;29(11):3740-3748.

7. Sadana A. Protein adsorption and inactivation on surfaces. Influence of heterogeneities. Chem Rev. 1992;92(8):1799-1818.

8. Linkov P, Artemyev M, Efimov AE, Nabiev I. Comparative advantages and limitations of the basic metrology methods applied to the characterization of nanomaterials. Nanoscale. 2013;5(19):8781-8798.

9. Salvador-Morales C, Valencia PM, Gao W, Karnik R, Farokhzad OC. Spontaneous formation of heterogeneous patches on polymer-lipid core-shell particle surfaces during self-assembly. Small. 2013;9(4):511-517.

10. Roh KH, Martin DC, Lahann J. Biphasic Janus particles with nanoscale anisotropy. Nat Mater. 2005;4(10):759-763.

11. Roh KH, Martin DC, Lahann J. Triphasic nanocolloids. J Am Chem Soc. 2006;128(21):6796-6797.

12. Mullen DG, Banaszak Holl MM. Heterogeneous ligand-nanoparticle distributions: a major obstacle to scientific understanding and commercial translation. Acc Chem Res. 2011;44(11):1135-1145. 
13. McPhail MR, Sells JA, He Z, Chusuei CC. Charging nanowalls: adjusting the carbon nanotube isoelectric point via surface functionalization. J Phys Chem C. 2009;113(32):14102-14109.

14. Florea I, Ersen O, Arenal R, et al. 3D analysis of the morphology and spatial distribution of nitrogen in nitrogen-doped carbon nanotubes by energy-filtered transmission electron microscopy tomography. $\mathrm{J} \mathrm{Am}$ Chem Soc. 2012;134(23):9672-9680.

15. RISE Microscopy: Correlative Raman SEM Imaging - WITec: Focus Innovations. Available from: http://www.witec.de/products/raman/ rise-microscopy/. Accessed July 3, 2014.

16. Heckel JC, Kisley LM, Mannion JM, Chumanov G. Synthesis and self-assembly of polymer and polymer-coated Ag nanoparticles by the reprecipitation of binary mixtures of polymers. Langmuir. 2009;25(17):9671-9676.

17. Shah AA, Schultz B, Kohlstedt KL, Glotzer SC, Solomon MJ. Synthesis, assembly, and image analysis of spheroidal patchy particles. Langmuir. 2013;29(15):4688-4696.

18. Arora S, Rajwade JM, Paknikar KM. Nanotoxicology and in vitro studies: the need of the hour. Toxicol Appl Pharmacol. 2012;258(2): $151-165$.

19. Reddy LH, Arias JL, Nicolas J, Couvreur P. Magnetic nanoparticles: design and characterization, toxicity and biocompatibility, pharmaceutical and biomedical applications. Chem Rev. 2012;112(11):5818-5878.

20. Valencia PM, Hanewich-Hollatz MH, Gao W, et al. Effects of ligands with different water solubilities on self-assembly and properties of targeted nanoparticles. Biomaterials. 2011;32(26):6226-6233.

21. Gomes R, Roming S, Przybilla A, Meier MAR, Feldmann C. Barium peroxide nanoparticles: synthesis, characterization and their use for actuating the luminol chemiluminescence. JMater Chem C. 2014;2(8): $1513-1518$

22. Kazarian SG, Chan KLA, Tay FH. ATR-FT-IR Imaging for Pharmaceutical and Polymeric Materials: From Micro to Macro Approaches. In: Salzer R, Siesler HW, editors. Infrared and Raman Spectroscopic Imaging. Weinheim: Wiley-VCH Verlag GmbH \& Co. KGaA; 2009:347-375.

23. Liu H, Webster TJ. Nanomedicine for implants: a review of studies and necessary experimental tools. Biomaterials. 2006;28(2):354-369.

24. Mudunkotuwa IA, Minshid AA, Grassian VH. ATR-FTIR spectroscopy as a tool to probe surface adsorption on nanoparticles at the liquid-solid interface in environmentally and biologically relevant media. Analyst. 2014;139(5):870-881.

25. Sundaram S, Sacksteder CA, Weber TJ, et al. Fourier-transform infrared spectroscopy for rapid screening and live-cell monitoring: application to nanotoxicology. Nanomedicine (Lond). 2013;8(1):145-156.

26. Cruz LJ, Tacken PJ, Fokkink R, Figdor CG. The influence of PEG chain length and targeting moiety on antibody-mediated delivery of nanoparticle vaccines to human dendritic cells. Biomaterials. 2011; 32(28):6791-6803.

27. Lin P, Lin CW, Mansour R, Gu F. Improving biocompatibility by surface modification techniques on implantable bioelectronics. Biosens Bioelectron. 2013;47:451-460.

28. Wu H, Zhu L, Torchilin VP. pH-sensitive poly(histidine)-PEG/DSPEPEG co-polymer micelles for cytosolic drug delivery. Biomaterials. 2013;34(4):1213-1222.

29. Fregnaux M, Gaumet JJ, Dalmasso S, Laurenti JP, Schneider R. Mass spectrometry techniques in the context of nanometrology. Microelectron Eng. 2013;108:187-191.

30. Bednar AJ, Poda AR, Mitrano DM, et al. Comparison of on-line detectors for field flow fractionation analysis of nanomaterials. Talanta. 2013;104:140-148.

31. Laborda F, Bolea E, Jiménez-Lamana J. Single particle inductively coupled plasma mass spectrometry: a powerful tool for nanoanalysis. Anal Chem. 2013;86(5):2270-2278.

32. Cedervall T, Lynch I, Lindman S, et al. Understanding the nanoparticleprotein corona using methods to quantify exchange rates and affinities of proteins for nanoparticles. Proc Natl Acad Sci U S A. 2007;104(7): 2050-2055.
33. Mochalov KE, Efimov AE, Bobrovsky A, et al. Combined scanning probe nanotomography and optical microspectroscopy: a correlative technique for 3D characterization of nanomaterials. ACS Nano. 2013; 7(10):8953-8962.

34. Baer DR, Gaspar DJ, Nachimuthu P, Techane SD, Castner DG. Application of surface chemical analysis tools for characterization of nanoparticles. Anal Bioanal Chem. 2010;396(3):983-1002.

35. Sitterberg J, Oezcetin A, Ehrhardt C, Bakowsky U. Utilising atomic force microscopy for the characterisation of nanoscale drug delivery systems. Eur J Pharm Biopharm. 2010;74(1):2-13.

36. PekarekKJ, Jacob JS, MathiowitzE. Double-walled polymer microspheres for controlled drug release. Nature. 1994;367(6460):258-260.

37. Xiao CD, Shen XC, Tao L. Modified emulsion solvent evaporation method for fabricating core-shell microspheres. Int J Pharm. 2013; 452(1-2):227-232.

38. Jin-Phillipp NY, Krauss TN, van Aken PA. The growth of one-dimensional $\mathrm{CuPcF} 16$ nanostructures on gold nanoparticles as studied by transmission electron microscopy tomography. ACS Nano. 2012;6(5):4039-4044

39. Sharma R, Bisen DP, Shukla U, Sharma BG. X-ray diffraction: a powerful method of characterizing nanomaterials. Recent Research in Science and Technology. 2012;4(8):77-79. Available from: http:// recent-science.com/index.php/rrst/article/viewFile/14887/7589. Accessed October 3, 2014.

40. Bóta A. Development of powder diffraction apparatus for smallangle X-ray scattering measurements. J Appl Crystallogr. 2013;46(2): 573-576.

41. Renaud G, Lazzari R, Leroy F. Probing surface and interface morphology with grazing incidence small angle X-ray scattering. Surf Sci Rep. 2009;64(8):255-380.

42. Gouadec G, Colomban P. Raman spectroscopy of nanostructures and nanosized materials. J Raman Spectrosc. 2007;38(6):598-603.

43. Kiefer W. Recent advances in linear and nonlinear Raman spectroscopy II. J Raman Spectrosc. 2008;39(12):1710-1725.

44. Arora AK, Rajalakshmi M, Ravindran TR, Sivasubramanian V. Raman spectroscopy of optical phonon confinement in nanostructured materials. J Raman Spectrosc. 2007;38(6):604-617.

45. Rolo AG, Vasilevskiy MI. Raman spectroscopy of optical phonons confined in semiconductor quantum dots and nanocrystals. $J$ Raman Spectrosc. 2007;38(6):618-633.

46. Bhaskar S, Gibson CT, Yoshida M, et al. Engineering, characterization and directional self-assembly of anisotropically modified nanocolloids. Small. 2011;7(6):812-819.

47. Llop J, Estrela-Lopis I, Ziolo RF, et al. Uptake, biological fate, and toxicity of metal oxide nanoparticles. Part Part Syst Char. 2014;31(1): 24-35.

48. Andrade N, Martinez D, Paula A, Silveira J, Alves O, Souza Filho A. Temperature effects on the nitric acid oxidation of industrial grade multiwalled carbon nanotubes. J Nanopart Res. 2013;15(7):1-11.

49. Haase A, Tentschert J, Jungnickel H, et al. Toxicity of silver nanoparticles in human macrophages: uptake, intracellular distribution and cellular responses. J Phys Conf Ser. 2011;304(1):012030.

50. Romero G, Estrela-Lopis I, Zhou J, et al. Surface engineered poly(lactide-co-glycolide) nanoparticles for intracellular delivery: uptake and cytotoxicity - a confocal Raman microscopic study. Biomacromolecules. 2010;11(11):2993-2999.

51. Buyens K, De Smedt SC, Braeckmans K, et al. Liposome based systems for systemic siRNA delivery: stability in blood sets the requirements for optimal carrier design. J Control Release. 2012;158(3): $362-370$.

52. Sugiyama I, Sadzuka Y. Correlation of fixed aqueous layer thickness around PEG-modified liposomes with in vivo efficacy of antitumor agent-containing liposomes. Curr Drug Discov Technol. 2011;8(4):357-366.

53. Allen C, Dos Santos N, Gallagher R, et al. Controlling the physical behavior and biological performance of liposome formulations through use of surface grafted poly(ethylene glycol). Biosci Rep. 2002;22(2):225-250. 
54. Salvador-Morales C, Zhang L, Langer R, Farokhzad OC. Immunocompatibility properties of lipid-polymer hybrid nanoparticles with heterogeneous surface functional groups. Biomaterials. 2009; 30(12):2231-2240.

55. Fukushima K, Hedrick JL, Nelson A, Sanders DP, inventors; International Business Machines Corporation, USA, assignee. Surface modified nanoparticles, methods of their preparation, and uses thereof for gene and drug delivery. US Patent US20110182996A12011.

56. Horcajada P, Chalati T, Serre C, et al. Porous metal-organic-framework nanoscale carriers as a potential platform for drug delivery and imaging. Nat Mater. 2010;9(2):172-178.

57. Horcajada Cortes P, Ferey G, Serre C, Gref R, Couvreur P, inventors; Centre National de la Recherche Scientifique - CNRS, Fr; Universite de Versailles Saint-Quentin-en-Yvelines, assignee. Organic-inorganic hybrid solid with a modified surface. US Patent WO2009077671A12009.

58. Bae Y, Kataoka K. Intelligent polymeric micelles from functional poly(ethylene glycol)-poly(amino acid) block copolymers. Adv Drug Deliv Rev. 2009;61(10):768-784.

59. Chiappetta DA, Sosnik A. Poly(ethylene oxide)-poly(propylene oxide) block copolymer micelles as drug delivery agents: improved hydrosolubility, stability and bioavailability of drugs. Eur J Pharm Biopharm. 2007;66(3):303-317.

60. Aliabadi HM, Lavasanifar A. Polymeric micelles for drug delivery. Expert Opin Drug Deliv. 2006;3(1):139-162.

61. Lukyanov AN, Torchilin VP. Micelles from lipid derivatives of watersoluble polymers as delivery systems for poorly soluble drugs. Adv Drug Deliv Rev. 2004;56(9):1273-1289.

62. Torchilin VP. PEG-based micelles as carriers of contrast agents for different imaging modalities. Adv Drug Deliv Rev. 2002;54(2) 235-252.

63. Lavasanifar A, Samuel J, Kwon GS. Poly(ethylene oxide)-blockpoly(L-amino acid) micelles for drug delivery. Adv Drug Deliv Rev. 2002;54(2):169-190.

64. Ren J, Shen S, Wang D, et al. The targeted delivery of anticancer drugs to brain glioma by PEGylated oxidized multi-walled carbon nanotubes modified with angiopep-2. Biomaterials. 2012;33(11):3324-3333.

65. Cheng J, Meziani MJ, Sun YP, Cheng SH. Poly(ethylene glycol)conjugated multi-walled carbon nanotubes as an efficient drug carrier for overcoming multidrug resistance. Toxicol Appl Pharmacol. 2011; 250(2):184-193.

66. Bhirde AA, Patel S, Sousa AA, et al. Distribution and clearance of PEG-single-walled carbon nanotube cancer drug delivery vehicles in mice. Nanomedicine (Lond). 2010;5(10):1535-1546.

67. Liu Z, Sun X, Nakayama-Ratchford N, Dai H. Supramolecular chemistry on water-soluble carbon nanotubes for drug loading and delivery. ACS Nano. 2007;1(1):50-56.

68. Angelini G, Boncompagni S, De Maria P, et al. Layer-by-layer deposition of shortened nanotubes or polyethylene glycol-derivatized nanotubes on liposomes: a tool for increasing liposome stability. Carbon. 2007;45(13):2479-2485.

69. Graham NB. Introduction to biotechnical and biomedical applications of poly(ethylene glycol). In: Harris JM, editor. Poly(Ethylene Glycol) Chemistry: Biotechnical and Biomedical Applications. New York, NY, USA: Plenum Press; 1992.

70. Mosqueira VC, Legrand P, Gulik A, et al. Relationship between complement activation, cellular uptake and surface physicochemical aspects of novel PEG-modified nanocapsules. Biomaterials. 2001;22(22):2967-2979.

71. Hamad I, Al-Hanbali O, Hunter AC, Rutt KJ, Andresen TL, Moghimi SM. Distinct polymer architecture mediates switching of complement activation pathways at the nanosphere-serum interface: implications for stealth nanoparticle engineering. ACS Nano. 2010;4(11):6629-6638.

72. Graham NB. Poly(ethylene glycol) gels and drug delivery. In: Harris JM, editor. Poly(Ethylene Glycol) Chemistry: Biotechnical and Biomedical Applications. New York, NY, USA: Plenum Press; 1992.
73. Gref R, Lück M, Quellec P, et al. 'Stealth' corona-core nanoparticles surface modified by polyethylene glycol (PEG): influences of the corona (PEG chain length and surface density) and of the core composition on phagocytic uptake and plasma protein adsorption. Colloids Surf B Biointerfaces. 2000;18(3-4):301-313.

74. Bailey FE Jr. Poly(ethylene oxide). Maryland Heights, MO, USA: Elsevier; 2012.

75. Deng L, Zhang Y, Ma L, et al. Comparison of anti-EGFR-Fab' conjugated immunoliposomes modified with two different conjugation linkers for siRNA delivery in SMMC-7721 cells. Int J Nanomedicine. 2012;8:3271-3283.

76. Hansen CM. Hansen Solubility Parameters: A User's Handbook. Boca Raton, FL, USA: CRC Press; 2012.

77. Rodriguez F, Cohen C, Ober CK, Archer LA. Viscous flow. In: Principles of Polymer Systems. 5th ed. New York, NY, USA: Taylor and Francis Books Inc; 2003.

78. Chan JM, Zhang L, Yuet KP, et al. PLGA-lecithin-PEG core-shell nanoparticles for controlled drug delivery. Biomaterials. 2009; 30(8):1627-1634.

79. Shi J, Xiao Z, Vilos C, Votruba A, Langer RS, Farokhzad OC. Lipidpolymer hybrid particles. Google Patents; 2011.

80. Zhang L, He Y, Ma G, Song C. [Preparation, characterization and in vitro and in vivo studies on folate-targeted biodegradable polymersomes loaded with paclitaxel]. Zhongguo Zhong Yao Za Zhi. 2010;45(22):1742-1748. Chinese.

81. Farokhzad OC, Salvador-Morales C, Gao W, Zhang L, Chen JM, Langer RS, inventors; Brigham and Women's Hospital Inc, USA; Massachusetts Institute of Technology, assignee. Particles with multiple functionalized surface domains for controlled and sustained drug release. US Patent WO2010042555A22010.

82. Feng G, Ding D, Li K, Liu J, Liu B. Reversible photoswitching conjugated polymer nanoparticles for cell and ex vivo tumor imaging. Nanoscale. 2014;6(8):4141-4147.

83. Riley T, Heald CR, Stolnik S, et al. Core-shell structure of PLAPEG nanoparticles used for drug delivery. Langmuir. 2003;19(20): 8428-8435.

84. Milani S, Bombelli FB, Pitek AS, Dawson KA, Rädler J. Reversible versus irreversible versus irreversible binding of transferrin to polystyrene nanoparticles: soft and hard corona. ACS Nano. 2012;6(3) 2532-2541.

85. Mahmoudi M, Saeedi-Eslami SN, Shokrgozar MA, et al. Cell "vision”: complementary factor of protein corona in nanotoxicology. Nanoscale. 2012;4(17):5461-5468.

86. Andersson J, Ekdahl KN, Lambris JD, Nilsson B. Binding of C3 fragments on top of adsorbed plasma proteins during complement activation on a model biomaterial surface. Biomaterials. 2005;26(13): 1477-1485.

87. Zeng S, Huang YM, Chang CE, Zhong W. Protein binding for detection of small changes on a nanoparticle surface. Analyst. 2014; 139(6):1364-1371.

88. Treuel L, Brandholt S, Maffre P, Wiegele S, Shang L, Nienhaus GU. Impact of protein modification on the protein corona on nanoparticles and nanoparticle-cell interactions. ACS Nano. 2014;8(1):503-513.

89. Naahidi S, Jafari M, Edalat F, Raymond K, Khademhosseini A, Chen P. Biocompatibility of engineered nanoparticles for drug delivery. J Control Release. 2013;166(2):182-194.

90. Wei A, Mehtala JG, Patri AK. Challenges and opportunities in the advancement of nanomedicines. J Control Release. 2012;164(2): 236-246.

91. Zasadzinski JA, Stenger PC, Shieh I, Dhar P. Overcoming rapid inactivation of lung surfactant: analogies between competitive adsorption and colloid stability. Biochim Biophys Acta. 2010;1798(4): 801-828.

92. Tcholakova S, Denkov ND, Sidzhakova D, Ivanov IB, Campbell B. Effects of electrolyte concentration and $\mathrm{pH}$ on the coalescence stability of $\beta$-lactoglobulin emulsions: experiment and interpretation. Langmuir. 2005;21(11):4842-4855. 
93. Kwon MJ, Lee J, Wark AW, Lee HJ. Nanoparticle-enhanced surface plasmon resonance detection of proteins at attomolar concentrations: comparing different nanoparticle shapes and sizes. Anal Chem. 2012;84(3):1702-1707.

94. Kristensen SH, Pedersen GA, Nejsum LN, Sutherland DS. Protein adsorption at nanopatterned surfaces studied by quartz crystal microbalance with dissipation and surface plasmon resonance. JPhys Chem B. 2013;117(36):10376-10383.

95. Ohtsuki C, Ichikawa Y, Shibata H, Kawachi G, Torimoto T, Ogata S. Sensing of protein adsorption with a porous bulk composite comprising silver nanoparticles deposited on hydroxyapatite. J Mater Sci Mater Med. 2010;21(4):1225-1232.

96. Moghimi SM, Hunter AC, Andresen TL. Factors controlling nanoparticle pharmacokinetics: an integrated analysis and perspective. Annu Rev Pharmacol Toxicol. 2012;52:481-503.

97. Pio R, Ajona D, Lambris JD. Complement inhibition in cancer therapy. Semin Immunol. 2013;25(1):54-64.

98. Mortimer GM, Butcher NJ, Musumeci AW, Deng ZJ, Martin DJ, Minchin RF. Cryptic epitopes of albumin determine mononuclear phagocyte system clearance of nanomaterials. ACS Nano. 2014;8(4): 3357-3366.

99. Arnida, Malugin A, Ghandehari H. Cellular uptake and toxicity of gold nanoparticles in prostate cancer cells: a comparative study of rods and spheres. J Appl Toxicol. 2010;30(3):212-217.

100. Wang HJ, Cao Y, Sun YY, et al. Is there an optimal topographical surface in nanoscale affecting protein adsorption and cell behaviors? J Nanopart Res. 2011;13(9):4201-4210.

101. Wang HJ, Sun YY, Cao Y, et al. Is there an optimal topographical surface in nano-scale affecting protein adsorption and cell behaviors? Part II. J Nanopart Res. 2012;14(5):1-10.

102. Gon S, Santore MM. Sensitivity of protein adsorption to architectural variations in a protein-resistant polymer brush containing engineered nanoscale adhesive sites. Langmuir. 2011;27(24):15083-15091.

103. Hentschel C, Wagner H, Smiatek J, et al. AFM-based force spectroscopy on polystyrene brushes: effect of brush thickness on protein adsorption. Langmuir. 2013;29(6):1850-1856.

104. Gessner A, Lieske A, Paulke BR, Muller RH. Influence of surface charge density on protein adsorption on polymeric nanoparticles: analysis by two-dimensional electrophoresis. Eur J Pharm Biopharm. 2002;54(2):165-170.

105. Gessner A, Waicz R, Lieske A, Paulke BR, Mader K, Muller RH. Nanoparticles with decreasing surface hydrophobicities: influence on plasma protein adsorption. Int J Pharm. 2000;196(2):245-249.

106. Lück M, Paulke BR, Schröder W, Blunk T, Müller RH. Analysis of plasma protein adsorption on polymeric nanoparticles with different surface characteristics. J Biomed Mater Res. 1998;39(3):478-485.

107. Paulke BR, Moeglich PM, Knippel E, Budde A, Nitzche R, Muller RH. Electrophoretic 3D-mobility profiles of latex particles with different surface groups. Langmuir. 1995;11(1):70-74.

108. Norde W. Surface-tethered polymers to influence protein adsorption and microbial adhesion. Z Phys Chem (N F). 2007;221(1):47-63.

109. Ma N, Ma C, Li C, et al. Influence of nanoparticle shape, size, and surface functionalization on cellular uptake. J Nanosci Nanotechnol. 2013;13(10):6485-6498.

110. Yezek L. Surfactant Adsorption on Colloids: Studies Using the Electrophoretic Fingerprinting Approach. In: Somasundaran, P, editor. Encyclopedia of Surface and Colloid Science. 2nd ed. 2006:6048-6055.

111. Walkey CD, Chan WC. Understanding and controlling the interaction of nanomaterials with proteins in a physiological environment. Chem Soc Rev. 2012;41(7):2780-2799.

112. Imaz A, Ramos J, Forcada J. Surface functionalization of latex particles. In: Hidalgo-Alvarez R, editor. Structure and Functional Properties of Colloidal Systems. Boca Raton, FL, USA: CRC Press; 2009.

113. Oehlke K, Adamiuk M, Behsnilian D, et al. Potential bioavailability enhancement of bioactive compounds using food-grade engineered nanomaterials: a review of the existing evidence. Food Funct. 2014;5(7):1341-1359.
114. Bancos S, Tyner KM, Weaver JL. Immunotoxicity testing for drug-nanoparticle conjugates: regulatory considerations. In: Dobrovolskaia MA, McNeil SE, editors. Handbook of Immunological Properties of Engineered Nanomaterials. Volume 1. Hackensack, NJ, USA: World Scientific Publishing Co; 2013.

115. Mérian J, Boisgard R, Decleves X, Thezé B, Texier I, Tavitian B. Synthetic lipid nanoparticles targeting steroid organs. J Nucl Med. 2013;54(11):1996-2003.

116. Singh S, Sharma A, Robertson GP. Realizing the clinical potential of cancer nanotechnology by minimizing toxicologic and targeted delivery concerns. Cancer Res. 2012;72(22):5663-5668.

117. Simkó M, Nosske D, Kreyling W. Metrics, dose, and dose concept: the need for a proper dose concept in the risk assessment of nanoparticles. Int J Environ Res Public Health. 2014;11(4):4026-4048.

118. Cohen JM, Teeguarden JG, Demokritou P. An integrated approach for the in vitro dosimetry of engineered nanomaterials. Part Fibre Toxicol. 2014;11(1):20.

119. Sauer UG, Vogel S, Aumann A, et al. Applicability of rat precisioncut lung slices in evaluating nanomaterial cytotoxicity, apoptosis, oxidative stress, and inflammation. Toxicol Appl Pharmacol. 2014;276(1):1-20.

120. Donaldson K, Schinwald A, Murphy F, et al. The biologically effective dose in inhalation nanotoxicology. Acc Chem Res. 2012;46(3): $723-732$.

121. Vernon JA, Golec JH, Dimasi JA. Drug development costs when financial risk is measured using the Fama-French three-factor model. Health Econ. 2010;19(8):1002-1005.

122. US Food and Drug Administration. Code of Federal Regulations Title 21. Silver Spring, MD, USA: US Department of Human and Health Services; 2013. Available from: http://www.accessdata.fda. gov/scripts/cdrh/cfdocs/cfcfr/CFRSearch.cfm?fr=56.122. Accessed October 3, 2014.

123. US Food and Drug Administration. Guidance for Industry: CGMP for Phase 1 Investigational Drugs. In: US Department of Human and Health Services. Rockville, MD, USA: Office of Training and Communication, Division of Drug information, Center for Drug Evaluation and Research, Food and Drug Administration; 2008. Available from: http://www.fda.gov/downloads/Drugs/GuidanceComplianceRegulator yInformation/Guidances/ucm070273.pdf. Accessed October 3, 2014.

124. Tyner K, Sadrieh N. Considerations when submitting nanotherapeutics to FDA/CDER for regulatory review. In: McNeil SE, editor. Characterization of Nanoparticles Intended for Drug Delivery. Totowa, NJ, USA: Humana Press; 2011.

125. Baraldi G, Carrada M, Toudert J, et al. Preventing the degradation of Ag nanoparticles using an ultrathin a- $\mathrm{Al}_{2} \mathrm{O}_{3}$ layer as protective barrier. J Phys Chem C. 2013;117(18):9431-9439.

126. Ramos J, Forcada J, Hidalgo-Alvarez R. Cationic polymer nanoparticles and nanogels: from synthesis to biotechnological applications. Chem Rev. 2014;114(1):367-428.

127. Richardson SD, Ternes TA. Water analysis: emerging contaminants and current issues. Anal Chem. 2014;86(6):2813-2848.

128. Zolnik BS, Sadrieh N. Regulatory perspective on the importance of ADME assessment of nanoscale material containing drugs. Adv Drug Deliv Rev. 2009;61(6):422-427.

129. Gebel T, Marchan R, Hengstler JG. The nanotoxicology revolution. Arch Toxicol. 2013;87(12):2057-2062.

130. Klaper R, Arndt D, Bozich J, Dominguez G. Molecular interactions of nanomaterials and organisms: defining biomarkers for toxicity and high-throughput screening using traditional and next-generation sequencing approaches. Analyst. 2014;139(5):882-895.

131. Setyawati MI, Fang W, Chia SL, Leong DT. Nanotoxicology of common metal oxide based nanomaterials: their ROS-y and non-ROS-y consequences. Asia-Pacific Journal of Chemical Engineering. 2013;8(2):205-217.

132. Paciotti GF, Myer L, Weinreich D, et al. Colloidal gold: a novel nanoparticle vector for tumor directed drug delivery. Drug Deliv. 2004;11(3):169-183. 
International Journal of Nanomedicine

Dovepress

\section{Publish your work in this journal}

The International Journal of Nanomedicine is an international, peerreviewed journal focusing on the application of nanotechnology in diagnostics, therapeutics, and drug delivery systems throughout the biomedical field. This journal is indexed on PubMed Central, MedLine, CAS, SciSearch ${ }^{\circledR}$, Current Contents ${ }^{\circledR} /$ Clinical Medicine,
Journal Citation Reports/Science Edition, EMBase, Scopus and the Elsevier Bibliographic databases. The manuscript management system is completely online and includes a very quick and fair peer-review system, which is all easy to use. Visit http://www.dovepress.com/ testimonials.php to read real quotes from published authors.

Submit your manuscript here: http://www.dovepress.com/international-journal-of-nanomedicine-journal 